\title{
Sensitivity of SARS-CoV-2 B.1.1.7 to mRNA vaccine-elicited antibodies
}

https://doi.org/10.1038/s41586-021-03412-7

Received: 26 January 2021

Accepted: 1 March 2021

Published online: 11 March 2021

Check for updates

\author{
Dami A. Collier, 1,3,144, Anna De Marco ${ }^{4,144}$, Isabella A. T. M. Ferreira ${ }^{1,2,144}$, Bo Meng ${ }^{1,2,144}$, \\ Rawlings P. Datir, ${ }^{1,2,3,44}$, Alexandra C. Walls ${ }^{5}$, Steven A. Kemp ${ }^{1,2,3}$, Jessica Bassi ${ }^{4}$, Dora Pinto ${ }^{4}$, \\ Chiara Silacci-Fregni ${ }^{4}$, Siro Bianchi ${ }^{4}$, M. Alejandra Tortorici ${ }^{5}$, John Bowen ${ }^{5}$, Katja Culap ${ }^{4}$, \\ Stefano Jaconi ${ }^{4}$, Elisabetta Cameroni ${ }^{4}$, Gyorgy Snell ${ }^{6}$, Matteo S. Pizzuto ${ }^{4}$, \\ Alessandra Franzetti Pellanda ${ }^{7}$, Christian Garzoni ${ }^{7}$, Agostino Riva ${ }^{8}$, The CITIID-NIHR BioResource \\ COVID-19 Collaboration*, Anne Elmer ${ }^{9}$, Nathalie Kingston ${ }^{10}$, Barbara Graves ${ }^{10}$, Laura E. McCoy ${ }^{3}$, \\ Kenneth G. C. Smith ${ }^{1,2}$, John R. Bradley ${ }^{2,10}$, Nigel Temperton ${ }^{11}$, Lourdes Ceron-Gutierrez ${ }^{12}$, \\ Gabriela Barcenas-Morales ${ }^{12,13}$, The COVID-19 Genomics UK (COG-UK) Consortium*, \\ William Harvey ${ }^{14}$, Herbert W. Virgin ${ }^{6}$, Antonio Lanzavecchia ${ }^{4}$, Luca Piccoli ${ }^{4}$, Rainer Doffinger ${ }^{12,15}$, \\ Mark Wills ${ }^{2}$, David Veesler ${ }^{5}$, Davide Corti ${ }^{4,145 \bowtie}$ \& Ravindra K. Gupta ${ }^{1,2,15,16,17,1,145 凶}$
}

Transmission of SARS-CoV-2 is uncontrolled in many parts of the world; control is compounded in some areas by the higher transmission potential of the B.1.1.7 variant ${ }^{1}$, which has now been reported in 94 countries. It is unclear whether the response of the virus to vaccines against SARS-CoV-2 on the basis of the prototypic strain will be affected by the mutations found in B.1.1.7. Here we assess the immune responses of individuals after vaccination with the mRNA-based vaccine BNT162b2 ${ }^{2}$. We measured neutralizing antibody responses after the first and second immunizations using pseudoviruses that expressed the wild-type spike protein or a mutated spike protein that contained the eight amino acid changes found in the B.1.1.7 variant. The sera from individuals who received the vaccine exhibited a broad range of neutralizing titres against the wild-type pseudoviruses that were modestly reduced against the B.1.1.7 variant. This reduction was also evident in sera from some patients who had recovered from COVID-19. Decreased neutralization of the B.1.1.7 variant was also observed for monoclonal antibodies that target the $\mathrm{N}$-terminal domain ( 9 out of 10 ) and the receptor-binding motif ( 5 out of 31 ), but not for monoclonal antibodies that recognize the receptor-binding domain that bind outside the receptor-binding motif. Introduction of the mutation that encodes the E484K substitution in the B.1.1.7 background to reflect a newly emerged variant of concern (VOC 202102/02) led to a more-substantial loss of neutralizing activity by vaccine-elicited antibodies and monoclonal antibodies (19 out of 31) compared with the loss of neutralizing activity conferred by the mutations in B.1.1.7 alone. The emergence of the E484K substitution in a B.1.1.7 background represents a threat to the efficacy of the BNT162b2 vaccine.
The BNT162b2 mRNA vaccine encodes the full-length trimerized spike protein of SARS CoV- $2^{2}$ and was designed against the Wuhan- 1 isolate. Concerns have been raised as to whether vaccines will be effective against newly emergent SARS-CoV-2 variants, such as B.1.1.7 (N501Y. $\mathrm{V} 1)^{3}$. In clinical studies of BNT162b2, the geometric mean titre (GMT) of neutralizing antibodies associated with $50 \%$ neutralization increased after the first dose and the vaccine provided high levels of protection against infection and severe disease after the second dose ${ }^{4}$.

\section{Activity of vaccine and convalescent sera against B.1.1.7} Participants $(n=37)$ received the first dose of the BNT162b2 mRNA vaccine 3 weeks before blood was drawn for the collection of serum and peripheral blood mononuclear cells. The median age was 62 years (interquartile range, $47-84$ years) and $35 \%$ of participants were female. Of these participants, 21 individuals also had a blood draw 3 weeks after receiving the second dose of the BNT162b2 mRNA vaccine. Serum IgG titres against nucleocapsid protein, the spike protein and the receptor-binding domain (RBD) of the spike protein were assayed (Extended Data Fig. 1a).

Using lentiviral pseudotyping, we studied the wild-type (wild-type spike bearing D614G) and mutant B.1.1.7 spike proteins (Fig. 1a) to measure the neutralization activity of vaccine-elicited sera. The vaccine sera exhibited a range of inhibitory dilutions that provided $50 \%$ neutralization $\left(\mathrm{ID}_{50}\right)$ (Fig. 1b, c). The GMT against the wild-type spike protein after the second dose of vaccine was substantially higher than after the first dose (318 compared with 77) (Fig. 1b, e). There was correlation between total 


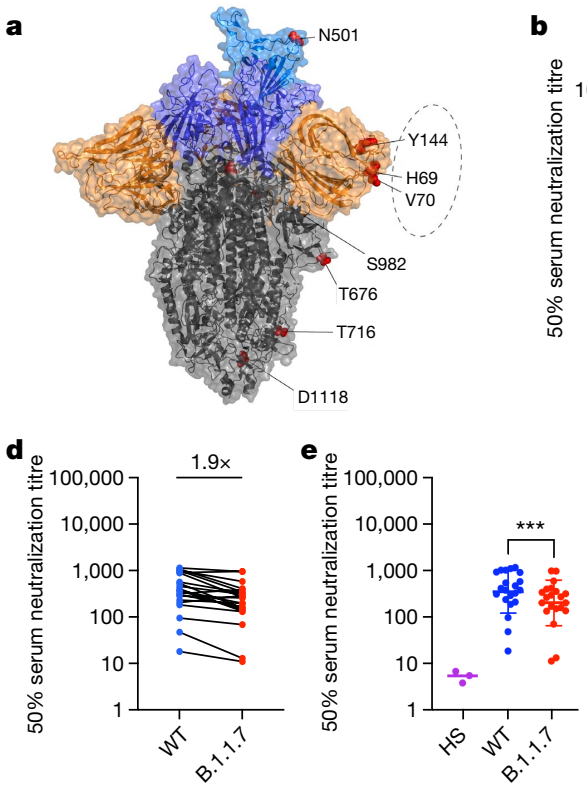

Fig. 1 Neutralization by sera from the first and second dose of the BNT162b2 mRNA vaccine against wild-type and B.1.1.7 spike mutant SARS-CoV2-pseudotyped viruses. a, Spike in the open conformation with a single erect RBD (Protein Data Bank (PDB): 6ZGG) is shown in the vertical view of the trimer axis. The locations of mutated residues are shown as red spheres, with deletions indicated in a dashed outline, and are labelled on the monomer with an erect RBD. b-g, The $50 \%$ serum neutralization titres of the first dose of the vaccine (b, c, $n=37)$, the second dose of the vaccine $(\mathbf{d}, \mathbf{e}, n=21)$ and convalescent sera

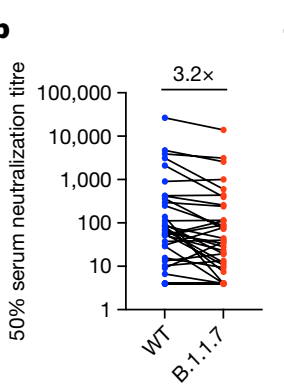

c
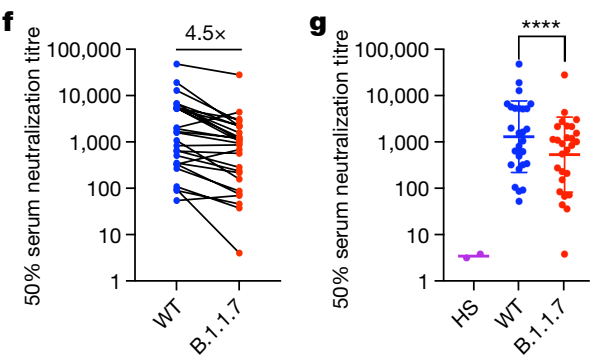

$(\mathbf{f}, \mathbf{g}, n=27)$ against the wild-type (WT) spike protein and the spike protein of the variant B.1.1.7 (containing the N501Y, A570D, $\Delta$ H69/DV70, $\triangle$ Y 144, P681H, T716I, S982A and D1118H mutations). HS, human serum control.b, d,f, Mean fold changes in $\mathrm{ID}_{50}$ are indicated above the graphs. Data points of the same individual are connected by lines. Data are GMT \pm s.d. and individual values of two independent experiments each with two technical repeats. Two-tailed Wilcoxon matched-pairs signed-rank test with no adjustment for multiple comparisons; ${ }^{* *} P<0.01,{ }^{* * * *} P<0.0001$. The cut-off for $50 \%$ neutralization was set to 4 . spike IgG titres and serum neutralization titres (Extended Data Fig. 1b). A broad range of T cell responses was measured by IFN $\gamma$ FluoroSpot against SARS-CoV-2 peptides in samples from individuals who received the vaccine after the first dose. These cellular responses did not correlate with serum neutralization titres or IgG spike antibody titres (Extended Data Fig.1c, d).

We then generated mutated pseudoviruses carrying the spike protein with the N501Y and A570D substitutions and the H69/V70 deletion $(\Delta \mathrm{H} 69 / \Delta V 70)$. We observed a small increase in the ability of sera from individuals who were vaccinated or had recovered from COVID-19 to inhibit this triple-mutant virus (Extended Data Fig. 2a-c). We next included the full set of eight mutations in the spike protein that is present in the B.1.1.7 variant (Fig. 1a). Of the 29 sera with neutralization activity after the first dose, 20 showed evidence of a reduction in neutralization titres against the B.1.1.7 variant (Fig. 1b, c and Extended Data Fig. 3), with a fold change of $3.2 \pm 5.7$ (mean \pm s.d.). After the second dose, the GMT was markedly increased compared with the first-dose titres, with a fold change of $1.9 \pm 0.9$ (mean \pm s.d.) (Fig. 1d, e). Among sera from 27 individuals who had recovered from COVID-19, the GMT at 50\% neutralization was 1,334 for the wild-type spike protein, which is significantly higher than the GMT after the second dose of the vaccine (Fig. 1f, g). The fold change in $I_{50}$ for neutralization of the B.1.1.7 compared with wild-type (D614G) spike protein was $4.5 \pm 8.7$ (Fig.1f, $g$ and Extended Data Fig. 4).

The E484K substitution (Fig. 2a) has been reported as an escape mutation for several monoclonal antibodies ${ }^{5}$, and is present in the B.1.351 (501Y.V2) and P.1 (501Y.V3) lineages. As of 11 February 2021, 30 B.1.1.7 sequences also had the E484K substitution (Fig. 2c). Phylogenetic analysis suggests that there have been multiple independent acquisitions, with one lineage appearing to expand over time, indicating active transmission (Fig. 2b). This has resulted in Public Health England naming this a variant of concern (VOC 202102/02) ${ }^{6}$. We therefore generated pseudoviruses that carried the B.1.1.7 spike mutations with or without the additional $\mathrm{E} 484 \mathrm{~K}$ substitution and tested these against sera obtained after the first and second dose of the BNT162b2 mRNA vaccine as well as against convalescent sera. After the second vaccine dose, we observed a considerable loss of neutralizing activity for the pseudovirus with the B.1.1.7 spike mutations and E484K (Fig. 3d, e). The mean fold change for the E484K-containing B.1.1.7 spike variant was 6.7 compared with 1.9 for the B.1.1.7 variant, relative to the wild-type spike protein (Fig. 3a-c and Extended Data Fig. 5). Similarly, when we tested a panel of convalescent sera with a range of neutralization titres (Fig. 1f, g and Extended Data Fig. 5), we observed additional loss of activity against the mutant B.1.1.7 spike with $\mathrm{E} 484 \mathrm{~K}$, with fold change of 11.4 relative to the wild-type spike protein (Fig. 3f, g and Extended Data Fig. 5).

\section{Monoclonal antibody activity against B.1.1.7}

We tested 60 monoclonal antibodies isolated from 15 individuals who had recovered from SARS-CoV-2 infection in early 2020 with an in vitro pseudotyped neutralization assay against the B.1.1.7 spike protein (Supplementary Table 1). Out of 60 monoclonal antibodies, 20 (33.3\%) showed a greater than twofold loss of neutralizing activity against the B.1.1.7 variant compared to wild-type SARS-CoV-2 (Fig. 4a, b and Extended Data Fig. 6). The B.1.1.7 mutant virus fully escaped neutralization by 8 out of 10 monoclonal antibodies (80\%) that target the $\mathrm{N}$-terminal domain (NTD) (Fig. 4c). Of the 31 monoclonal antibodies that target the receptor-binding motif (RBM), 5 (16.1\%) showed more than 100-fold decrease in B.1.1.7 neutralization, and additional 6 monoclonal antibodies (19.4\%) had a partial 2-10-fold reduction (Fig. 4d). Finally, all RBD-specific non-RBM-targeting monoclonal antibodies that were tested fully retained neutralizing activity against B.1.1.7 (Fig. 4e).

To address the role of the N501Y substitution in B.1.1.7 in the neutralization escape from RBM-specific antibodies, we tested the binding of 50 RBD-specific monoclonal antibodies to the wild-type and N501Y-mutant RBD by biolayer interferometry (Fig. 4 fand Extended Data Fig. 7). The 5 RBM-specific monoclonal antibodies that did not neutralize the B.1.1.7 variant (Fig. 4d) showed a complete loss of binding to the N501Y-mutant RBD (Fig. 4g, h), demonstrating a role for this mutation as an escape mechanism for certain RBM-targeting monoclonal antibodies. 


\section{a}

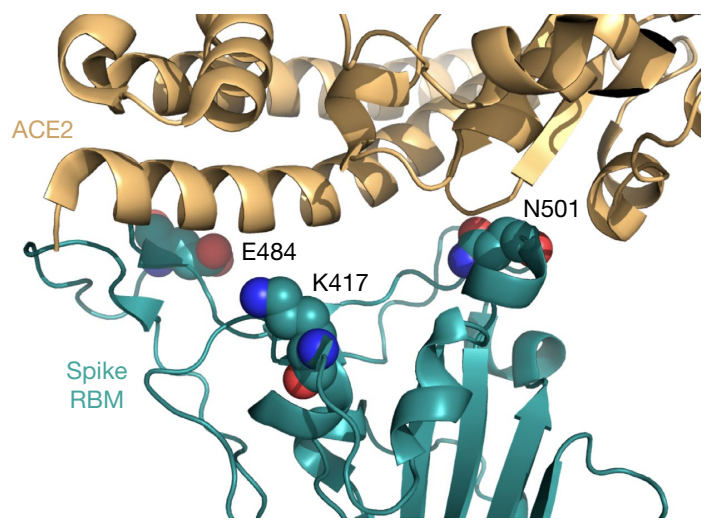

c

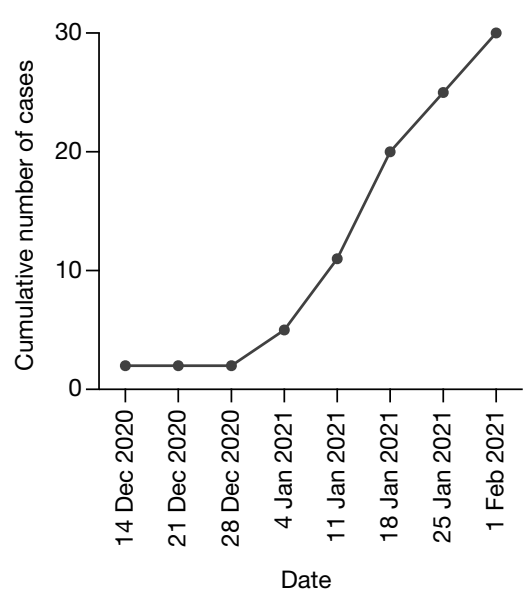

b

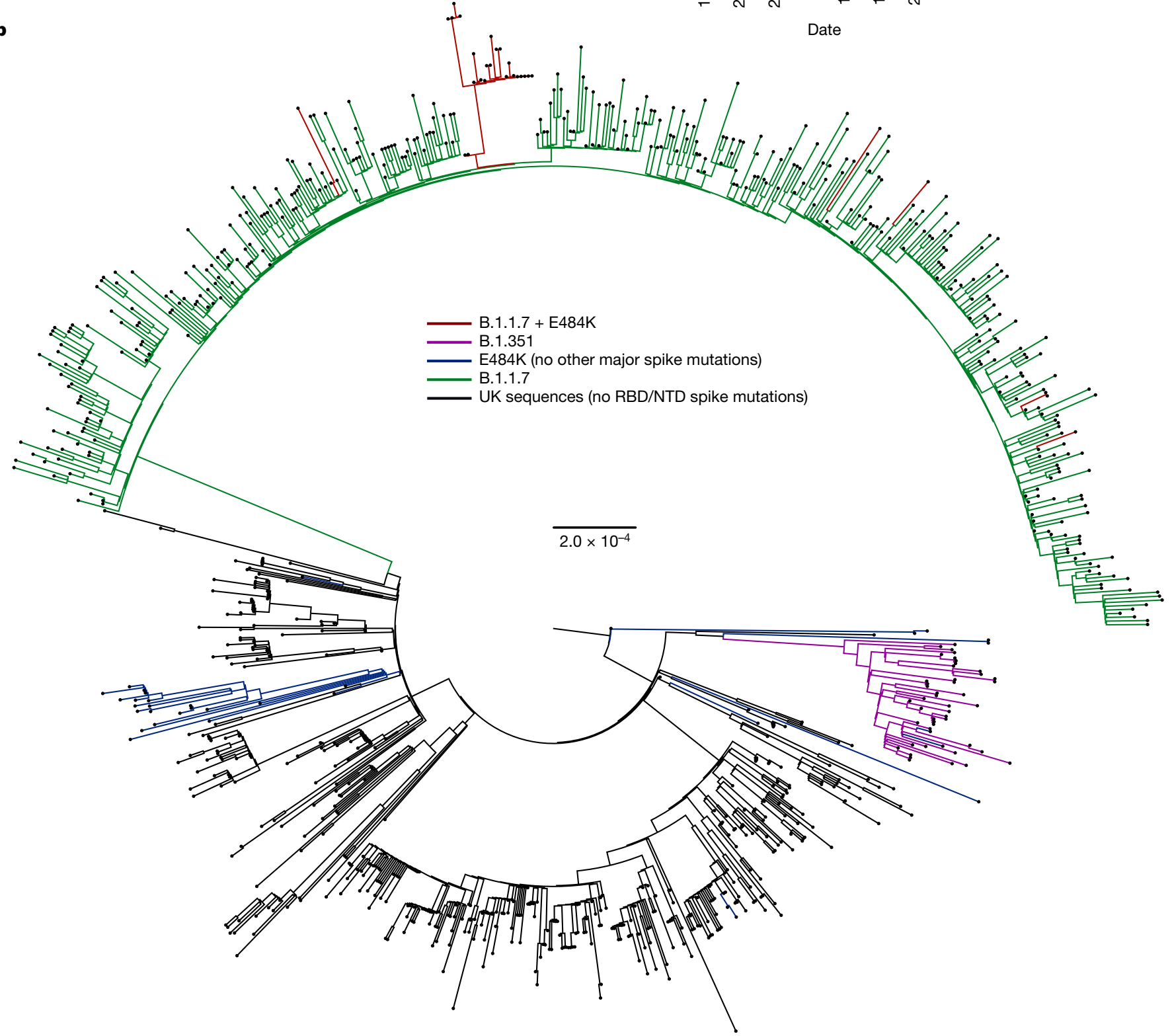

Fig. 2 | The E484K substitution was found in the background of B.1.1.7 and showed evidence of transmission. a, Representation of the spike RBM:ACE2 interface (PDB: 6MOJ) with residues E484, N501 and K417 highlighted as spheres coloured by element. b, Maximum likelihood phylogeny of a subset of sequences from the UK with the E484K mutation (blue) and the B.1.1.7 lineage

To assess the effect of $\mathrm{E} 484 \mathrm{~K}$ on this panel of monoclonal antibodies, we generated a triple-mutant SARS-CoV-2 pseudotype virus carrying the K417N, E484K and N501Y mutations (spike(N501Y, E484K, K417N)). (green), with background sequences from the UK without RBD mutations shown in black. As of 11 February 2021, 30 sequences from the B.1.1.7 lineage (one cluster of 25 at the top of the phylogenetic tree) have acquired the $\mathrm{E} 484 \mathrm{~K}$ substitution (red).c, Sequence accumulation over time in GISAID for UK sequences of the B.1.1.7 and other variants with or without E484K.

The inclusion of the $\mathrm{K} 417 \mathrm{~N}$ substitution was prompted by the observation that substitutions at this position have been found in five sequences from recent viral isolates within the B.1.1.7 lineage (K417 to 
a

$\rightarrow \mathrm{WT} \rightarrow \mathrm{B} \cdot 1 \cdot 1.7-\mathrm{B} \cdot 1 \cdot 1 \cdot 7+\mathrm{E} 484 \mathrm{~K}$
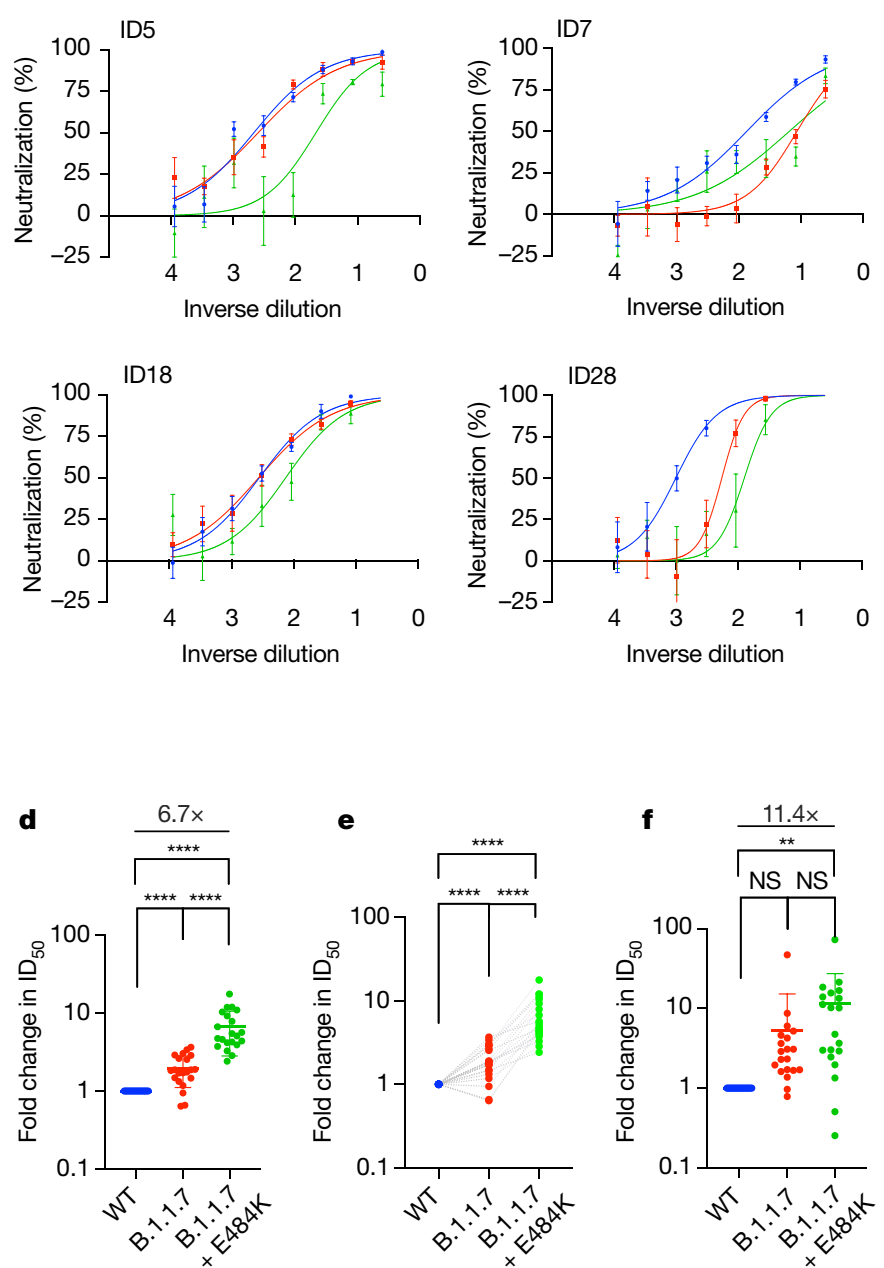

Asn, Glu or Arg). This is in keeping with the convergent evolution of the virus to an RBD containing N501Y, E484K and K417N or K417T as evidenced by the B.1.351 and P.1 lineages. Notably, mutations at K417 are reported to escape neutralization by monoclonal antibodies, including the recently approved monoclonal antibody LY-CoV016 ${ }^{5,7}$. Out of the 60 monoclonal antibodies tested, 20 (33.3\%) showed a loss of neutralizing activity against the spike(N501Y, E484K, K417N) mutant of more than 10-fold compared to wild-type SARS-CoV-2 (Fig. 4a, b and Extended Data Fig. 6), and of these 19 are RBM-specific monoclonal antibodies. As above, we addressed the role of the $\mathrm{E} 484 \mathrm{~K}$ substitution in the escape from RBM-specific antibodies by testing the binding of 50 RBD-specific monoclonal antibodies to the RBD of the wild-type and E484K-mutant spike protein by biolayer interferometry (Fig. $4 \mathrm{f}$ and Extended Data Fig. 8). Out of the 19RBM-specific monoclonal antibodies that showed reduced or loss of neutralization of the spike(N501Y, E484K, K417N) mutant (Fig. 4d), 16 showed a complete or partial loss of binding to the RBD of the E484K mutant (Fig. $4 \mathrm{~g}$, h), which is consistent with findings that $\mathrm{E} 484 \mathrm{~K}$ is an important mutation for viral escape $\mathrm{e}^{8-10}$. In addition, 3 of these 16 monoclonal antibodies also lost the ability to bind to an RBD containing the N501Y substitution, indicating that a fraction of RBM-specific antibodies are sensitive to both the N501Y and E484K substitutions. Similarly, 3 of the 19 monoclonal antibodies that lost neutralization against the spike(N501Y, E484K, K417N) mutant (S2D8, S2H7 and S2X128) were previously shown to lose binding and neutralization to the K417V mutant, and are here shown to be sensitive to either the N501Y or the E484K substitution.
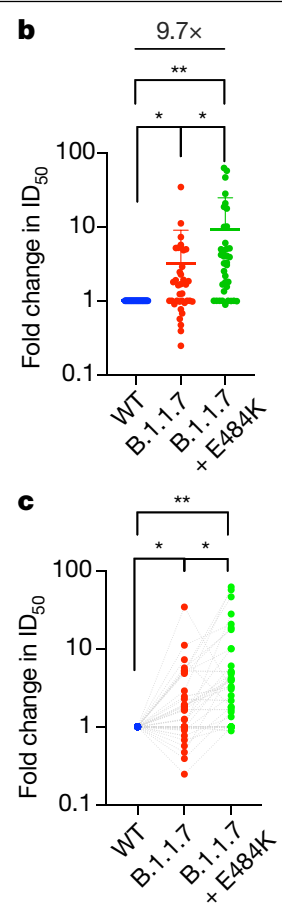

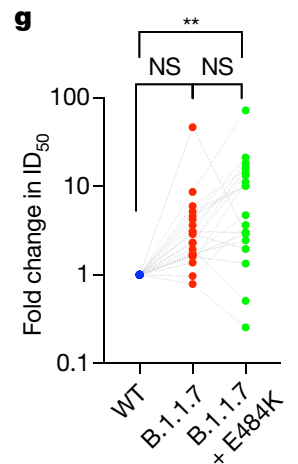

Fig. 3 | Neutralization potency of mRNA vaccine sera and convalescent sera (before SARS-CoV-2 B.1.1.7) against a pseudotyped virus with the spike mutations of the B1.1.7 lineage with or without E484K. All virus variants were in a spike(D614G) background. a, Example neutralization curves of vaccinated individuals (ID $5,7,18,28)$. The inverse dilution is shown on a log scale. Data are mean \pm s.e.m. representative of two independent experiments each with two technical replicates. b-g, The $50 \%$ neutralization titres of each virus against sera derived after the first vaccine dose (b, c, $n=37)$, the second vaccine dose (d, e, $n=21)$ and for convalescent sera (f, $\mathbf{g}, n=20$ ) expressed as fold change relative to the wild-type virus. b, $\mathbf{d}, \mathbf{f}$, Mean fold changes in $\mathrm{ID}_{50}$ are indicated above the graphs. Data are mean \pm s.d. and individual values; error bars for negative values are not shown.c,e, $\mathbf{g}$, Data are the mean fold change of two technical replicates and are representative of two independent experiments. Data points of the same individual are connected by lines. b, d,f, Two-tailed paired Student's $t$-test; ${ }^{*} P<0.05$, ${ }^{* *} P<0.01,{ }^{* * * *} P<0.0001$; NS, not significant. The cut-off for $50 \%$ neutralization was set to 4 .

\section{Binding of the RBD of the B.1.1.7 variant to ACE2}

Using biolayer interferometry, we found that human ACE2 bound to the RBD of the B.1.1.7 variant with an affinity of $22 \mathrm{nM}$ compared to an affinity of $133 \mathrm{nM}$ for the wild-type RBD (Extended Data Fig. 9), in agreement with our previous deep-mutational scanning measurements using dimeric ACE2 ${ }^{11}$. Although ACE2 bound with comparable on rates to both RBDs, the observed dissociation rate constant was slower for B.1.1.7 than for the wild-type RBD (Extended Data Table1). These findings could explain the efficient ongoing transmission of this newly emergent SARS-CoV-2 lineage and the possibly reduced opportunity for antibody binding. To understand the effect of the mutations in the triple mutant $(\mathrm{K} 417 \mathrm{~N}$, E484K and N501Y), we evaluated the binding of ACE2 to the immobilized RBD of spike(N501Y, E484K, K417N). We determined an ACE2-binding affinity of $64 \mathrm{nM}$ for the RBD of spike(N501Y, E484K, K417N), driven by a faster off rate than observed for the RBD of the B.1.1.7 variant but slower than for the wild-type RBD. We propose that the K417N mutation is slightly detrimental to ACE2 binding, which explains the intermediate affinity determined for the RBD of spike(N501Y, E484K, K417N) compared to the B.1.1.7 and wild-type RBDs, probably as a result of disrupting the salt bridge formed with ACE2 residue D30.

\section{Discussion}

Serum neutralizing activity is a correlate of protection for other respiratory viruses, including influenza ${ }^{12}$ and respiratory syncytial virus, for which 


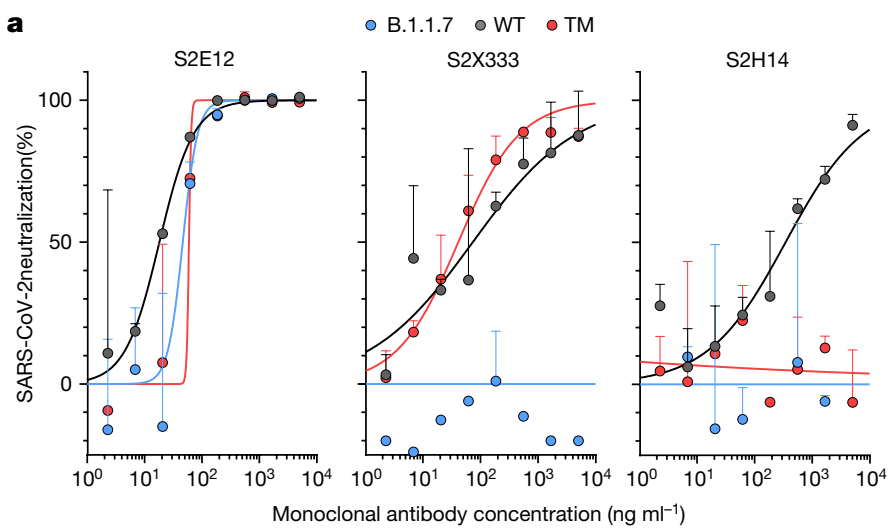

b

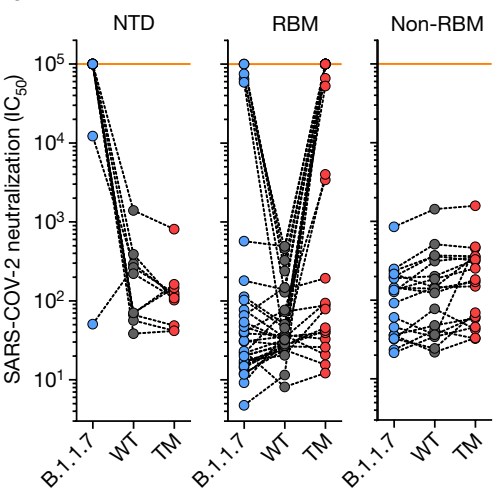

- B.1.1.7 ० WT ० TM

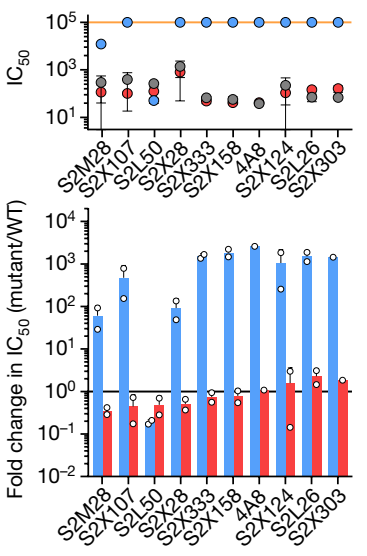

e
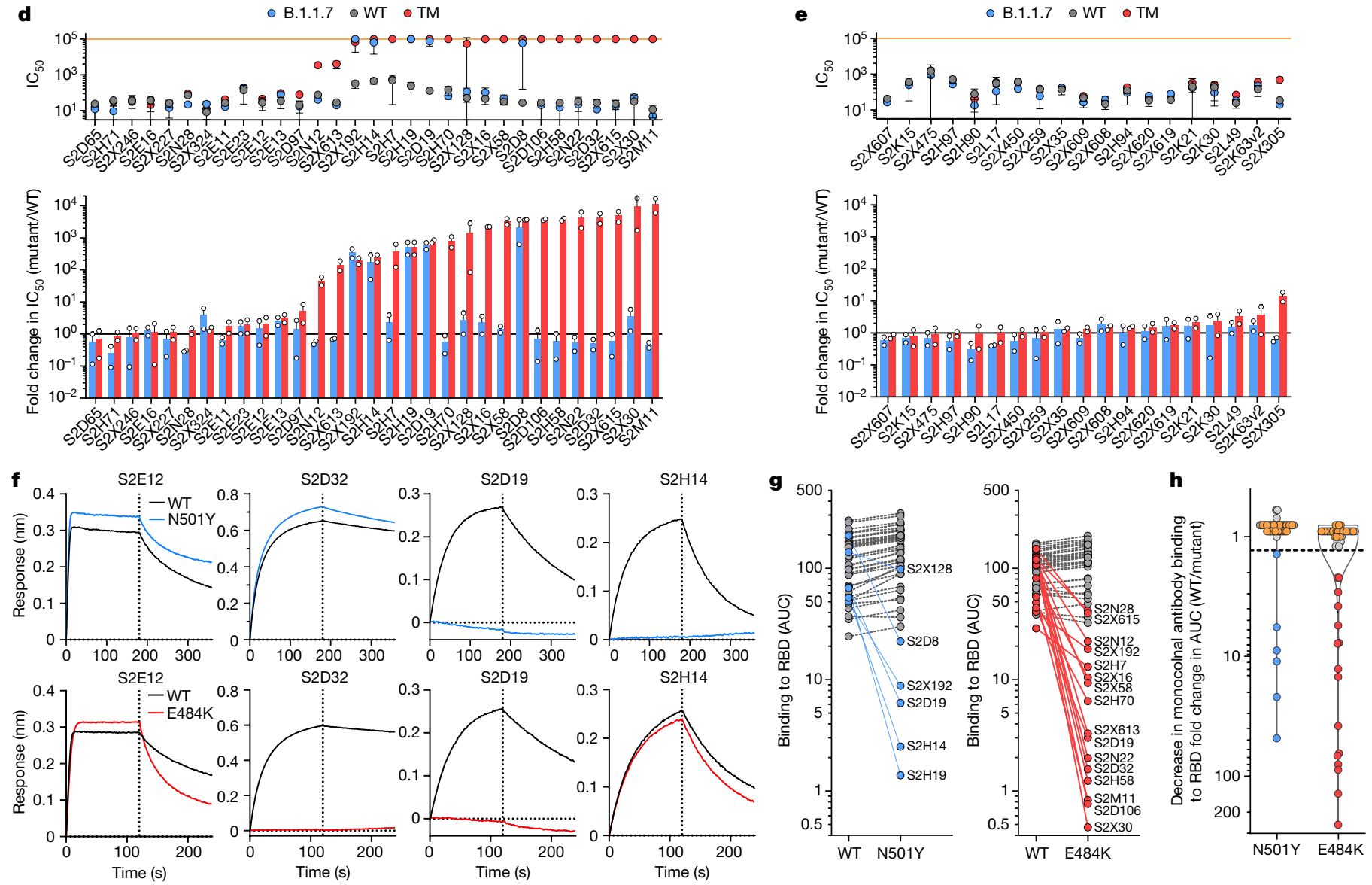

Fig. 4 | Neutralization and binding by a panel of NTD- and RBD-specific monoclonal antibodies against wild-type, B.1.1.7 and RBD-mutant SARS-CoV-2 viruses. a, Neutralization of pseudotyped SARS-CoV-2-murine leukemia virus (MLV) carrying wild-type spike (spike(D614G)) (grey), spike from B.1.1.7 (blue) or a triple-mutant spike protein (TM, carrying RBD mutations K417N, E484K and N501Y) (red) by three selected monoclonal antibodies (S2E12, S2X333 and S2H14) from one representative experiment. Data are mean \pm s.d. of two technical replicates. b, Neutralization of SARS-CoV-2-MLVs carrying wild-type spike (spike(D614G)), spike from B.1.1.7 or a triple-mutant spike protein (spike(N501Y, E484K, K417N)) by 60 monoclonal antibodies targeting the NTD $(n=10), \operatorname{RBM}(n=31)$ or non-RBM sites in the $\operatorname{RBD}(n=19)$. Data are the mean $50 \%$ inhibitory concentration $\left(\mathrm{IC}_{50}\right)$ values $\left(\mathrm{ng} \mathrm{ml}^{-1}\right)$ of $n=2$ independent experiments. c-e, Neutralization by NTD-specific (c),

RBM-specific (d) and non-RBM-specific (e) monoclonal antibodies is shown as

prohylaxis with monoclonal antibodies has been used in at-riskgroups ${ }^{13,14}$. Neutralizing antibody titres seemed to be highly correlated with vaccine protection against SARS-CoV-2 rechallenge in nonhuman primates ${ }^{15,16}$. the mean IC ${ }_{50}$ values (top) and mean fold change in B.1.1.7 (blue) or the triple mutant (spike(N501Y, E484K, K417N)) (red) relative to the wild-type virus (bottom). The orange line shows the threshold for non-neutralizing titres. Top, data are mean \pm s.d. IC $_{50}$ values from two independent experiments. Bottom, data are mean \pm s.d. fold change from two independent experiments. $\mathbf{f}-\mathbf{h}$, The kinetics of the binding of monoclonal antibodies to wild-type (black), N501Y (blue) and E484K (red) RBD as measured by biolayer interferometry. $f$, The four RBM-targeting monoclonal antibodies with no reduced binding to the RBD with N501Y or E484K are shown. g, h, Area under the curve (AUC) (g) and the fold change in the area under the curve (h) of 50 monoclonal antibodies tested against the wild-type, N501Y and E484K RBD. Monoclonal antibodies with a more than 1.3-fold (cut-off indicated by the orange line) change in area under the curve are shown in blue and red; orange dots show non-RBM-specific monoclonal antibodies.

This study reports on neutralization by sera collected after both the first and second doses of the BNT162b2 vaccine. The participants of this study were older adults, in line with the targeting of this age 
group in the initial rollout of the vaccination campaign in the UK. We demonstrate that neutralization of a pseudovirus containing the spike protein with the full set of mutations that is present in the B.1.1.7 variant showed a small reduction using sera from individuals who received the BNT162b2 vaccine that was more marked after the first dose than the second dose. This could be related to the increased breadth, potency and/or concentration of antibodies after the boost dose. Other studies have reported a small reduction in neutralization against the B.1.1.7 variant in individuals vaccinated with two doses of BNT162b $2{ }^{17}$ and mRNA-1273 ${ }^{18}$. The reduced neutralizing activity observed with polyclonal antibodies elicited by mRNA vaccines observed in this study is further supported by the loss of neutralizing activity observed with human monoclonal antibodies directed against both the RBD and, to a major extent, the NTD.

Multiple variants, including the 501Y.V2 and B.1.1.7 lineages, have multiple mutations as well as deletions in the NTD, most of which are located in a site of vulnerability that is targeted by all known NTD-specific neutralizing antibodies ${ }^{19,20}$. The role of NTD-specific neutralizing antibodies might be underestimated, in part by the use of neutralization assays based on target cells that overexpress ACE2 receptors. NTD-specific monoclonal antibodies were suggested to interfere with viral entry based on other accessory receptors, such as DC-SIGN and L-SIGN ${ }^{21}$, and their neutralization potency was found to be dependent on different in vitro culture conditions ${ }^{19}$. The observation that 9 out of 10 NTD-specific neutralizing antibodies did not show a complete or near-complete loss of neutralizing activity against B.1.1.7 indicates that this new variant may have also evolved to escape from this class of antibodies, which may have a yet unrecognized role in protective immunity. Taken together, the presence of multiple escape mutations in the NTD is supportive of the hypothesis that this region of the spike, in addition to the RBM, is also under immune pressure.

Worryingly, we have shown that there are multiple B.1.1.7 sequences in the UK that contain the E484K substitution with early evidence of transmission as well as independent acquisitions. We measured a further reduction in neutralization titres by vaccine sera when $\mathrm{E} 484 \mathrm{~K}$ was present alongside the B.1.1.7 spike mutations. A recent study ${ }^{18}$ has also shown that variants carrying the $\mathrm{E} 484 \mathrm{~K}$ substitution resulted in a 3-6-fold reduction in neutralization by sera from individuals who received the mRNA-1273 vaccine. Consistently, in this study we found that approximately $50 \%$ of the RBM-specific monoclonal antibodies tested lost neutralizing activity against SARS-CoV-2 carrying E484K. E484K has been shown to affect neutralization by monoclonal antibodies or convalescent sera, especially in combination with N501Y and $\mathrm{K} 417 \mathrm{~N}^{8,22-24}$.

Vaccines are a key part of a long-term strategy to bring SARS-CoV-2 transmission under control. Our data suggest that vaccine escape by the virus of current spike-directed vaccines designed against the Wuhan-1 strain will be inevitable, particularly given that $\mathrm{E} 484 \mathrm{~K}$ is emerging independently and recurrently on a B.1.1.7 (501Y.V1) background, and given the rapid global spread of B.1.1.7. Other major variants with E484K such as 501Y.V2 and V3 are also spreading regionally. This should be mitigated by designing next-generation vaccines with mutated spike sequences and using alternative viral antigens.

\section{Online content}

Any methods, additional references, Nature Research reporting summaries, source data, extended data, supplementary information, acknowledgements, peer review information; details of author contributions and competing interests; and statements of data and code availability are available at https://doi.org/10.1038/s41586-021-03412-7.
1. Volz, E. et al. Transmission of SARS-CoV-2 lineage B.1.1.7 in England: insights from linking epidemiological and genetic data. Preprint at https://doi.org/10.1101/2020.12.30.20249034 (2021).

2. Mulligan, M. J. et al. Phase $\mathrm{I} / \mathrm{II}$ study of COVID-19 RNA vaccine BNT162b1 in adults. Nature 586, 589-593 (2020).

3. Kemp, S. A. et al. Recurrent emergence and transmission of a SARS-CoV-2 spike deletion H69/V7O. Preprint at https://doi.org/10.1101/2020.12.14.422555 (2021).

4. Polack, F. P. et al. Safety and efficacy of the BNT162b2 mRNA COVID-19 vaccine. N. Engl. J. Med. 383, 2603-2615 (2020).

5. Wang, P. et al. Antibody resistance of SARS-CoV-2 variants B.1.351 and B.1.1.7. Nature https://doi.org/10.1038/s41586-021-03398-2 (2021).

6. Public Health England. PHE statement on Variant of Concern and new Variant Under Investigation. https://www.gov.uk/government/news/phe-statement-on-variant-ofconcern-and-new-variant-under-investigation (2021).

7. Thomson, E. C. et al. Circulating SARS-CoV-2 spike N439K variants maintain fitness while evading antibody-mediated immunity. Cell 184, 1171-1187 (2021).

8. Greaney, A. J. et al. Comprehensive mapping of mutations in the SARS-CoV-2 receptor-binding domain that affect recognition by polyclonal human plasma antibodies. Cell Host Microbe https://doi.org/10.1016/j.chom.2021.02.003 (2021).

9. Andreano, E. et al. SARS-CoV-2 escape in vitro from a highly neutralizing COVID-19 convalescent plasma. Preprint at https://doi.org/10.1101/2020.12.28.424451 (2020).

10. Kalyaanamoorthy, S., Minh, B. Q., Wong, T. K. F., von Haeseler, A. \& Jermiin, L. S ModelFinder: fast model selection for accurate phylogenetic estimates. Nat. Methods 14, 587-589 (2017).

11. Starr, T. N. et al. Deep mutational scanning of SARS-CoV-2 receptor binding domain reveals constraints on folding and ACE2 binding. Cell 182, 1295-1310 (2020).

12. Verschoor, C. P. et al. Microneutralization assay titres correlate with protection against seasonal influenza H1N1 and H3N2 in children. PLOS ONE 10, e0131531 (2015).

13. Kulkarni, P. S., Hurwitz, J. L., Simões, E. A. F. \& Piedra, P. A. Establishing correlates of protection for vaccine development: considerations for the respiratory syncytial virus vaccine field. Viral Immunol. 31, 195-203 (2018).

14. Goddard, N. L., Cooke, M. C., Gupta, R. K. \& Nguyen-Van-Tam, J. S. Timing of monoclonal antibody for seasonal RSV prophylaxis in the United Kingdom. Epidemiol. Infect. 135, 159-162 (2007).

15. Mercado, N. B. et al. Single-shot Ad26 vaccine protects against SARS-CoV-2 in rhesus macaques. Nature 586, 583-588 (2020).

16. McMahan, K. et al. Correlates of protection against SARS-CoV-2 in rhesus macaques. Nature 590, 630-634 (2021).

17. Muik, A. et al. Neutralization of SARS-CoV-2 lineage B.1.1.7 pseudovirus by BNT162b2 vaccine-elicited human sera. Science https://doi.org/10.1126/science.abg6105 (2021).

18. $W u, K$. et al. mRNA-1273 vaccine induces neutralizing antibodies against spike mutants from global SARS-CoV-2 variants. Preprint at https://doi.org/10.1101/2021.01.25.427948 (2021).

19. McCallum, M. et al. $\mathrm{N}$-terminal domain antigenic mapping reveals a site of vulnerability for SARS-CoV-2. Preprint at https://doi.org/10.1101/2021.01.14.426475 (2021).

20. Suryadevara, N. et al. Neutralizing and protective human monoclonal antibodies recognizing the $\mathrm{N}$-terminal domain of the SARS-CoV-2 spike protein. Preprint at https:// doi.org/10.1101/2021.01.19.427324 (2021).

21. Soh, W. T. et al. The N-terminal domain of spike glycoprotein mediates SARS-CoV-2 infection by associating with L-SIGN and DC-SIGN. Preprint at https://doi.org/10.1101/ 2020.11.05.369264 (2020).

22. Tegally, H. et al. Detection of a SARS-CoV-2 variant of concern in South Africa. Nature https://doi.org/10.1038/s41586-021-03402-9 (2021).

23. Greaney, A. J. et al. Complete mapping of mutations to the SARS-CoV-2 spike receptorbinding domain that escape antibody recognition. Cell Host Microbe 29, 44-57 (2020).

24. Weisblum, Y. et al. Escape from neutralizing antibodies by SARS-CoV-2 spike protein variants. eLife 9, e61312 (2020).

Publisher's note Springer Nature remains neutral with regard to jurisdictional claims in published maps and institutional affiliations.

(c) The Author(s), under exclusive licence to Springer Nature Limited 2021, corrected publication 2022

${ }^{1}$ Cambridge Institute of Therapeutic Immunology \& Infectious Disease, Cambridge, UK. ${ }^{2}$ Department of Medicine, University of Cambridge, Cambridge, UK. ${ }^{3}$ Division of Infection and Immunity, University College London, London, UK. ${ }^{4}$ Humabs Biomed SA, a subsidiary of Vir Biotechnology, Bellinzona, Switzerland. ${ }^{5}$ Department of Biochemistry, University of Washington, Seattle, WA, USA. ${ }^{6}$ Vir Biotechnology, San Francisco, CA, USA. ${ }^{7}$ Clinic of Internal Medicine and Infectious Diseases, Clinica Luganese Moncucco, Lugano, Switzerland. ${ }^{8}$ Division of Infectious Diseases, Luigi Sacco Hospital, University of Milan, Milan, Italy. ${ }^{9} \mathrm{NIHR}$ Cambridge Clinical Research Facility, Cambridge, UK. ${ }^{10}$ NIHR Bioresource, Cambridge, UK. ${ }^{11}$ University of Kent, Canturbury, UK. ${ }^{12}$ Department of Clinical Biochemistry and Immunology, Addenbrooke's Hospital, Cambridge, UK. ${ }^{13}$ Laboratorio de Inmunologia, UNAM, Cuautitlán, Mexico. ${ }^{14}$ Institute of Biodiversity, University of Glasgow, Glasgow, UK. ${ }^{15}$ Department of Haematology, University of Cambridge, Cambridge, UK. ${ }^{16}$ University of KwaZulu Natal, Durban, South Africa. ${ }^{17}$ Africa Health Research Institute, Durban, South Africa. ${ }^{18}$ Department of Infectious Diseases, Cambridge University Hospitals NHS Trust, Cambridge, UK. ${ }^{144}$ These authors contributed equally: Dami A. Collier, Anna De Marco, Isabella A. T. M. Ferreira, Bo Meng, Rawlings P. Datir. ${ }^{145}$ These authors jointly supervised this work: Davide Corti, Ravindra K. Gupta. *Lists of authors and their affiliations appear online. ${ }^{{ }^{e}}$-mail: dcorti@vir.bio; rkg20@cam.ac.uk 


\section{The CITIID-NIHR BioResource COVID-19 Collaboration}

\section{Principal investigators}

Stephen Baker ${ }^{2,3}$, Gordon Dougan ${ }^{2,3}$, Christoph Hess ${ }^{2,319,20}$, Nathalie Kingston ${ }^{11,15}$, Paul J. Lehner ${ }^{2,3}$, Paul A. Lyons ${ }^{2,3}$, Nicholas J. Matheson ${ }^{2,3}$, Willem H. Owehand ${ }^{15}$ Caroline Saunders ${ }^{21}$, Charlotte Summers ${ }^{3,22,23,24}$, James E. D. Thaventhiran ${ }^{2,3,25}$, Mark Toshner ${ }^{3,22,23}$ \& Michael P. Weekes ${ }^{2}$

\section{CRF \& volunteer research nurses}

Ashlea Bucke ${ }^{21}$, Jo Calder ${ }^{21}$, Laura Canna ${ }^{21}$, Jason Domingo ${ }^{21}$, Anne Elmer ${ }^{21}$, Stewart Fuller ${ }^{21}$, Julie Harris ${ }^{26}$, Sarah Hewitt ${ }^{21}$, Jane Kennet ${ }^{21}$, Sherly Jose ${ }^{21}$, Jenny Kourampa ${ }^{21}$, Anne Meadows ${ }^{21}$, Criona O'Brien ${ }^{26}$, Jane Price ${ }^{21}$, Cherry Publico ${ }^{21}$, Rebecca Rastall ${ }^{21}$, Carla Ribeiro ${ }^{21}$, Jane Rowlands ${ }^{21}$, Valentina Ruffolo ${ }^{21} \&$ Hugo Tordesillas ${ }^{21}$

\section{Sample logistics}

Ben Bullman ${ }^{2}$, Benjamin J. Dunmore ${ }^{3}$, Stuart Fawke ${ }^{27}$, Stefan Gräf ${ }^{3,11,15}$, Josh Hodgson ${ }^{3}$,

Christopher Huang ${ }^{3}$, Kelvin Hunter ${ }^{2,3}$, Emma Jones $^{28}$, Ekaterina Legchenko ${ }^{3}$, Cecilia Matara ${ }^{3}$ Jennifer Martin ${ }^{3}$, Federica Mescia ${ }^{2,3}$, Ciara O'Donnell ${ }^{3}$, Linda Pointon ${ }^{3}$, Nicole Pond ${ }^{2,3}$, Joy Shih ${ }^{3}$, Rachel Sutcliffe ${ }^{3}$, Tobias Tilly ${ }^{3}$, Carmen Treacy ${ }^{3}$, Zhen Tong ${ }^{3}$, Jennifer Wood ${ }^{3} \&$ Marta Wylot $^{29}$

Sample processing \& data acquisition

Laura Bergamaschi ${ }^{2,3}$, Ariana Betancourt ${ }^{2,3}$, Georgie Bower ${ }^{2,3}$, Chiara Cossetti ${ }^{2,3}$, Aloka De Sa $^{3}$, Madeline Epping ${ }^{2,3}$, Stuart Fawke ${ }^{27}$, Richard Grenfell ${ }^{30}$, Andrew Hinch ${ }^{2,3}$, Oisin Huhn ${ }^{31}$, Sarah Jackson ${ }^{3}$, Isobel Jarvis ${ }^{3}$, Daniel Lewis ${ }^{3}$, Joe Marsden ${ }^{3}$, Francesca Nice ${ }^{32}$,

Georgina Okecha ${ }^{3}$, Ommar Omarjee ${ }^{3}$, Marianne Perera ${ }^{3}$, Nathan Richoz ${ }^{3}$,

Veronika Romashova ${ }^{2,3}$, Natalia Savinykh Yarkoni ${ }^{3}$, Rahul Sharma ${ }^{3}$, Luca Stefanucci ${ }^{15}$,

Jonathan Stephens ${ }^{15}$, Mateusz Strezlecki ${ }^{30} \&$ Lori Turner $^{2,3}$

Clinical data collection

Eckart M. D. D. De Bie ${ }^{3}$, Katherine Bunclark ${ }^{3}$, Masa Josipovic ${ }^{33}$, Michael Mackay ${ }^{3}$,

Federica Mescia ${ }^{2,3}$, Sabrina Rossi ${ }^{34}$, Mayurun Selvan ${ }^{3}$, Sarah Spencer $^{35}$ \& Cissy Yong ${ }^{34}$

Royal Papworth Hospital ICU

Ali Ansaripour ${ }^{23}$, Alice Michael ${ }^{23}$, Lucy Mwaura ${ }^{23}$, Caroline Patterson ${ }^{23} \&$ Gary Polwarth ${ }^{23}$

Addenbrooke's Hospital ICU

Petra Polgarova ${ }^{24}$ \& Giovanni di Stefano ${ }^{24}$

Cambridge \& Peterborough Foundation Trust

Codie Fahey ${ }^{36}$ \& Rachel Michel ${ }^{36}$

ANPC \& Centre for Molecular Medicine \& Innovative Therapeutics

Sze-How Bong ${ }^{37}$, Jerome D. Coudert ${ }^{38}$ \& Elaine Holmes ${ }^{39}$

\section{NIHR BioResource}

John Allison ${ }^{11,15}$, Helen Butcher ${ }^{11,40}$, Daniela Caputo ${ }^{11,40}$, Debbie Clapham-Riley ${ }^{11,40}$

Eleanor Dewhurst ${ }^{11,40}$, Anita Furlong ${ }^{11,40}$, Barbara Graves ${ }^{11,40}$, Jennifer Gray ${ }^{11,40}$, Tasmin Ivers ${ }^{11,40}$

Mary Kasanicki ${ }^{11,24}$, Emma Le Gresley ${ }^{11,40}$, Rachel Linger ${ }^{11,40}$, Sarah Meloy ${ }^{11,40}$,

Francesca Muldoon ${ }^{11,40}$, Nigel Ovington ${ }^{11,15}$, Sofia Papadia ${ }^{11,40}$, Isabel Phelan ${ }^{11,40}$

Hannah Stark ${ }^{11,40}$, Kathleen E. Stirrups ${ }^{11,15}$, Paul Townsend ${ }^{11,15}$, Neil Walker ${ }^{11,15}$ \&

Jennifer Webster ${ }^{11,40}$

${ }^{19}$ Department of Biomedicine, University Basel and University Hospital Basel, Basel,

Switzerland. ${ }^{20}$ Botnar Research Centre for Child Health (BRCCH), University Basel \& ETH

Zurich, Basel, Switzerland. ${ }^{21}$ Cambridge Clinical Research Centre, NIHR Clinical Research

Facility, Cambridge University Hospitals NHS Foundation Trust, Addenbrooke's Hospital,

Cambridge, UK. ${ }^{22}$ Heart and Lung Research Institute, Cambridge, UK. ${ }^{23}$ Royal Papworth

Hospital NHS Foundation Trust, Cambridge, UK. ${ }^{24}$ Addenbrooke's Hospital, Cambridge, UK

${ }^{25} \mathrm{MRC}$ Toxicology Unit, School of Biological Sciences, University of Cambridge, Cambridge,

UK. ${ }^{26}$ Department of Medicine, Cardiff University, Cardiff, UK. ${ }^{27}$ Cambridge Institute for

Medical Research, Cambridge, UK. ${ }^{28}$ Department of Veterinary Medicine, Cambridge, UK.

${ }^{29}$ Department of Biochemistry, University of Cambridge, Cambridge, UK. ${ }^{30}$ Cancer Research

UK, Cambridge Institute, University of Cambridge, Cambridge, UK. ${ }^{31}$ Department of

Obstetrics \& Gynaecology, The Rosie Maternity Hospital, Cambridge, UK. ${ }^{32}$ Centre for Enzyme

Innovation, University of Portsmouth (PORT), Portsmouth, UK. ${ }^{33}$ Institute of Microbiology and

Infection, University of Birmingham, Birmingham, UK. ${ }^{34}$ Department of Surgery,

Addenbrooke's Hospital, Cambridge, UK. ${ }^{35}$ Biochemistry and Molecular Genetics, University

of Colorado School of Medicine, Aurora, CO, USA. ${ }^{36} \mathrm{Cambridge}$ and Peterborough

Foundation Trust, Fulbourn Hospital, Fulbourn, UK. ${ }^{37}$ Australian National Phenome Centre

Murdoch University, Murdoch, Western Australia, Australia. ${ }^{38}$ Centre for Molecular Medicine

and Innovative Therapeutics, Health Futures Institute, Murdoch University, Perth, Western

Australia, Australia. ${ }^{39}$ Centre of Computational and Systems Medicine, Health Futures

Institute, Murdoch University, Perth, Western Australia, Australia. ${ }^{40}$ Department of Public

Health and Primary Care, School of Clinical Medicine, University of Cambridge,

Cambridge, UK

The COVID-19 Genomics UK (COG-UK) Consortium

Funding acquisition, leadership \& supervision, metadata curation, project administration, samples \& logistics, sequencing \& analysis, software \& analysis tools and visualization

Samuel C. Robson ${ }^{32}$
Funding acquisition, leadership \& supervision, metadata curation, project administration, samples \& logistics, sequencing \& analysis and software \& analysis tools

Nicholas J. Loman ${ }^{33} \&$ Thomas R. Connor ${ }^{26,41}$

Leadership \& supervision, metadata curation, project administration, samples \& logistics, sequencing \& analysis, software \& analysis tools and visualization

Tanya Golubchik ${ }^{42}$

Funding acquisition, metadata curation, samples \& logistics, sequencing \& analysis, software \& analysis tools and visualization

Rocio T. Martinez Nunez ${ }^{43}$

Funding acquisition, leadership \& supervision, metadata curation, project administration and samples \& logistics

Catherine Ludden ${ }^{2}$

Funding acquisition, leadership \& supervision, metadata curation, samples \& logistics and sequencing \& analysis

Sally Corden ${ }^{41}$

Funding acquisition, leadership \& supervision, project administration, samples \& logistics and sequencing $\&$ analysis

lan Johnston ${ }^{44}$ \& David Bonsall ${ }^{42}$

Funding acquisition, leadership \& supervision, sequencing \& analysis, software \& analysis tools and visualization

Colin P. Smith ${ }^{45}$ \& Ali R. Awan ${ }^{46}$

Funding acquisition, samples \& logistics, sequencing \& analysis, software \& analysis tools and visualization

Giselda Bucca ${ }^{45}$

Leadership \& supervision, metadata curation, project administration, samples \& logistics and sequencing $\&$ analysis

M. Estee Torok Th, $^{3,48}$

Leadership \& supervision, metadata curation, project administration, samples \& logistics and visualization

Kordo Saeed ${ }^{49,50}$ \& Jacqui A. Prieto ${ }^{49,51}$

Leadership \& supervision, metadata curation, project administration, sequencing \& analysis and software \& analysis tools

David K. Jackson ${ }^{44}$

Metadata curation, project administration, samples \& logistics, sequencing \& analysis and software \& analysis tools

William L. Hamilton ${ }^{47,48}$

Metadata curation, project administration, samples \& logistics, sequencing \& analysis and visualization

Luke B. Snell ${ }^{52}$

Funding acquisition, leadership \& supervision, metadata curation and samples \& logistics Catherine Moore ${ }^{41}$

Funding acquisition, leadership \& supervision, project administration and samples \& logistics

Ewan M. Harrison ${ }^{2,44}$

Leadership \& supervision, metadata curation, project administration and samples \& logistics

Sonia Goncalves ${ }^{44}$

Leadership \& supervision, metadata curation, samples \& logistics and sequencing \& analysis

Derek J. Fairley ${ }^{53,54}$, Matthew W. Loose ${ }^{55}$ \& Joanne Watkins ${ }^{41}$

Leadership \& supervision, metadata curation, samples \& logistics and software \& analysis tools Rich Livett $^{44}$

Leadership \& supervision, metadata curation, samples \& logistics and visualization Samuel Moses ${ }^{56,57}$

Leadership \& supervision, metadata curation, sequencing \& analysis and software \& analysis tools

Roberto Amato ${ }^{44}$, Sam Nicholls ${ }^{33} \&$ Matthew Bull ${ }^{41}$

Leadership \& supervision, project administration, samples \& logistics and sequencing \& analysis

Darren L. Smith ${ }^{58,59,60}$ 
Leadership \& supervision, sequencing \& analysis, software \& analysis tools and visualization Jeff Barrett ${ }^{44} \&$ David M. Aanensen ${ }^{6}$

Metadata curation, project administration, samples \& logistics and sequencing \& analysis Martin D. Curran ${ }^{62}$, Surendra Parmar ${ }^{62}$, Dinesh Aggarwal ${ }^{2,44,63}$ \& James G. Shepherd ${ }^{64}$

Metadata curation, project administration, sequencing \& analysis and software \& analysis tools Matthew D. Parker ${ }^{65}$

Metadata curation, samples \& logistics, sequencing \& analysis and visualization Sharon Glaysher ${ }^{66}$

Metadata curation, sequencing $\&$ analysis, software $\&$ analysis tools and visualization Matthew Bashton ${ }^{58,59}$, Anthony P. Underwood ${ }^{61}$, Nicole Pacchiarinii ${ }^{41}$ \& Katie F. Loveson ${ }^{67}$

Project administration, sequencing \& analysis, software \& analysis tools and visualization Alessandro M. Carabelli²

Funding acquisition, leadership \& supervision and metadata curation Kate E. Templeton ${ }^{68,69}$

Funding acquisition, leadership \& supervision and project administration Cordelia F. Langford ${ }^{44}$, John Sillitoe ${ }^{44}$, Thushan I. de Silva ${ }^{65}$ \& Dennis Wang ${ }^{65}$

Funding acquisition, leadership \& supervision and sequencing \& analysis Dominic Kwiatkowski ${ }^{44,70}$, Andrew Rambaut ${ }^{69}$, Justin O'Grady ${ }^{71,72} \&$ Simon Cottrell ${ }^{4}$

Leadership \& supervision, metadata curation and sequencing \& analysis Matthew T. G. Holden ${ }^{73}$ \& Emma C. Thomson ${ }^{64}$

Leadership \& supervision, project administration and samples \& logistics Husam Osman ${ }^{63,74}$, Monique Andersson ${ }^{75}$, Anoop J. Chauhan ${ }^{66}$ \& Mohammed O. Hassan-Ibrahim ${ }^{76}$

Leadership \& supervision, project administration and sequencing \& analysis Mara Lawniczak ${ }^{4}$

Leadership \& supervision, samples \& logistics and sequencing \& analysis Alex Alderton $^{44}$, Meera Chand ${ }^{63}$, Chrystala Constantinidou ${ }^{77}$, Meera Unnikrishnan ${ }^{77}$, Alistair C. Darby ${ }^{78}$, Julian A. Hiscox ${ }^{78} \&$ Steve Paterson ${ }^{78}$

Leadership \& supervision, sequencing \& analysis and software \& analysis tools Inigo Martincorena ${ }^{44}$, David L. Robertson ${ }^{64}$, Erik M. Volz ${ }^{79}$, Andrew J. Page ${ }^{71} \&$ Oliver G. Pybus ${ }^{80}$

Leadership \& supervision, sequencing \& analysis and visualization Andrew R. Bassett ${ }^{44}$

Metadata curation, project administration and samples \& logistics Cristina V. Ariani ${ }^{44}$, Michael H. Spencer Chapman ${ }^{2,44}$, Kathy K. Li ${ }^{64}$, Rajiv N. Shah ${ }^{64}$ Natasha G. Jesudason ${ }^{64}$ \& Yusri Taha ${ }^{81}$

Metadata curation, project administration and sequencing \& analysis Martin P. McHugh ${ }^{68}$ \& Rebecca Dewar ${ }^{68}$

Metadata curation, samples \& logistics and sequencing \& analysis Aminu S. Jahun ${ }^{82}$, Claire McMurray ${ }^{33}$, Sarojini Pandey ${ }^{83}$, James P. McKenna ${ }^{53}$, Andrew Nelson ${ }^{59,60}$, Gregory R. Young ${ }^{58,59}$, Clare M. McCann ${ }^{59,60}$ \& Scott Elliott ${ }^{66}$

\section{Metadata curation, samples \& logistics and visualization}

Hannah Lowe ${ }^{56}$

Metadata curation, sequencing \& analysis and software \& analysis tools Ben Temperton ${ }^{84}$, Sunando Roy ${ }^{85}$, Anna Price ${ }^{26}$, Sara Rey ${ }^{41}$ \& Matthew Wyles ${ }^{65}$

Metadata curation, sequencing \& analysis and visualization

Stefan Rooke ${ }^{69} \&$ Sharif Shaaban ${ }^{73}$

Project administration, samples \& logistics and sequencing \& analysis Mariateresa de Cesare ${ }^{86}$

Project administration, samples \& logistics and software \& analysis tools Laura Letchford ${ }^{44}$

Project administration, samples \& logistics and visualization Siona Silveira ${ }^{49}$, Emanuela Pelosi ${ }^{49}$ \& Eleri Wilson-Davies ${ }^{49}$
Sequencing \& analysis, software \& analysis tools and visualization Áine $\mathrm{O}^{\prime}$ Toole $^{69}$, Andrew R. Hesketh ${ }^{45}$, Richard Stark ${ }^{77}$, Louis du Plessis ${ }^{80}$, Chris Ruis ${ }^{2}$, Helen Adams ${ }^{87}$ \& Yann Bourgeois ${ }^{88}$

Funding acquisition \& leadership and supervision Stephen L. Michell ${ }^{84}$, Dimitris Grammatopoulos ${ }^{83,89}$, Jonathan Edgeworth ${ }^{90}$, Judith Breuer ${ }^{85,91}$ John A. Todd ${ }^{86}$ \& Christophe Fraser ${ }^{42}$

Funding acquisition and project administration David Buck ${ }^{86}$ \& Michaela John ${ }^{92}$

Leadership \& supervision and metadata curation Gemma L. Kay ${ }^{71}$

Leadership \& supervision and project administration Steve Palmer ${ }^{44}$, Sharon J. Peacock ${ }^{2,63}$ \& David Heyburn ${ }^{41}$

Leadership \& supervision and samples \& logistics

Danni Weldon ${ }^{44}$, Esther Robinson ${ }^{63,74}$, Alan McNally ${ }^{33,93}$, Peter Muir ${ }^{63}$, lan B. Vipond ${ }^{63}$, John Boyes ${ }^{94}$, Venkat Sivaprakasam ${ }^{95}$, Tranprit Salluja ${ }^{96}$, Samir Dervisevic ${ }^{97}$ \& Emma J. Meader ${ }^{97}$

Leadership \& supervision and sequencing \& analysis

Naomi R. Park ${ }^{44}$, Karen Oliver ${ }^{44}$, Aaron R. Jeffries ${ }^{84}$, Sascha Ott ${ }^{77}$, Ana da Silva Filipe ${ }^{64}$, David A. Simpson ${ }^{54} \&$ Chris Williams ${ }^{41}$

Leadership \& supervision and visualization Jane A. H. Masoli 84,98

Metadata curation and samples \& logistics

Bridget A. Knight ${ }^{84,98}$, Christopher R. Jones ${ }^{84,98}$, Cherian Koshy ${ }^{99}$, Amy Ash ${ }^{99}$, Anna Casey ${ }^{100}$ Andrew Bosworth ${ }^{63,74}$, Liz Ratcliffe ${ }^{100}$, Li Xu-McCrae ${ }^{74}$, Hannah M. Pymont ${ }^{63}$

Stephanie Hutchings ${ }^{63}$, Lisa Berry ${ }^{83}$, Katie Jones $^{83}$, Fenella Halstead ${ }^{95}$, Thomas Davis ${ }^{101}$ Christopher Holmes ${ }^{102}$, Miren Iturriza-Gomara ${ }^{78}$, Anita O. Lucaci ${ }^{78}$, Paul Anthony Randell ${ }^{103,104}$ Alison Cox ${ }^{103,104}$, Pinglawathee Madona ${ }^{103,104}$, Kathryn Ann Harris ${ }^{91}$, Julianne Rose Brown ${ }^{91}$ Tabitha W. Mahungu ${ }^{105}$, Dianne Irish-Tavares ${ }^{105}$, Tanzina Haque ${ }^{105}$, Jennifer Hart ${ }^{105}$, Eric Witele ${ }^{105}$, Melisa Louise Fenton ${ }^{96}$, Steven Liggett ${ }^{106}$, Clive Graham ${ }^{107}$, Emma Swindells $^{108}$, Jennifer Collins ${ }^{81}$, Gary Eltringham ${ }^{81}$, Sharon Campbell ${ }^{109}$, Patrick C. McClure ${ }^{110}$ Gemma Clark ${ }^{111}$, Tim J. Sloan ${ }^{112}$, Carl Jones ${ }^{111}$ \& Jessica Lynch ${ }^{113,114}$

Metadata curation and sequencing \& analysis

Ben Warne ${ }^{115}$, Steven Leonard ${ }^{44}$, Jillian Durham ${ }^{44}$, Thomas Williams ${ }^{69}$, Sam T. Haldenby ${ }^{78}$ Nathaniel Storey ${ }^{91}$, Nabil-Fareed Alikhan ${ }^{71}$, Nadine Holmes ${ }^{55}$, Christopher Moore ${ }^{55}$, Matthew Carlile ${ }^{55}$, Malorie Perry ${ }^{41}$, Noel Craine ${ }^{41}$, Ronan A. Lyons ${ }^{116}$, Angela H. Beckett ${ }^{32}$, Salman Goudarzi ${ }^{67}$, Christopher Fearn ${ }^{67}$, Kate Cook $^{67}$, Hannah Dent ${ }^{67}$ \& Hannah Paul ${ }^{67}$

Metadata curation and software \& analysis tools

Robert Davies ${ }^{44}$

Project administration and samples \& logistics

Beth Blane ${ }^{2}$, Sophia T. Girgis ${ }^{2}$, Mathew A. Beale ${ }^{44}$, Katherine L. Bellis ${ }^{2,44}$, Matthew J. Dorman ${ }^{44}$, Eleanor Drury ${ }^{44}$, Leanne Kane ${ }^{44}$, Sally Kay ${ }^{44}$, Samantha McGuigan ${ }^{44}$, Rachel Nelson ${ }^{44}$, Liam Prestwood ${ }^{44}$, Shavanthi Rajatileka ${ }^{44}$, Rahul Batra ${ }^{90}$, Rachel J. Williams ${ }^{85}$ Mark Kristiansen ${ }^{85}$, Angie Green ${ }^{86}$, Anita Justice ${ }^{75}$, Adhyana I. K. Mahanama ${ }^{49,117}$ \& Buddhini Samaraweera ${ }^{49,117}$

Project administration and sequencing \& analysis Nazreen F. Hadjirin ${ }^{2} \&$ Joshua Quick ${ }^{33}$

Project administration and software \& analysis tools Radoslaw Poplawski ${ }^{33}$

Samples \& logistics and sequencing \& analysis

Leanne M. Kermack ${ }^{2}$, Nicola Reynolds ${ }^{118}$, Grant Hall ${ }^{82}$, Yasmin Chaudhry ${ }^{82}$, Malte L. Pinckert ${ }^{82}$ Iliana Georgana ${ }^{82}$, Robin J. Moll ${ }^{44}$, Alicia Thornton ${ }^{63}$, Richard Myers ${ }^{63}$, Joanne Stockton ${ }^{33}$, Charlotte A. Williams ${ }^{85}$, Wen C. Yew ${ }^{59}$, Alexander J. Trotter ${ }^{71}$, Amy Trebes ${ }^{86}$ George MacIntyre-Cockett ${ }^{86}$, Alec Birchley ${ }^{41}$, Alexander Adams ${ }^{41}$, Amy Plimmer ${ }^{41}$, Bree Gatica-Wilcox ${ }^{41}$, Caoimhe McKerr ${ }^{41}$, Ember Hilvers ${ }^{41}$, Hannah Jones ${ }^{41}$, Hibo Ásad ${ }^{41}$, Jason Coombes ${ }^{41}$, Johnathan M. Evans ${ }^{41}$, Laia Fina ${ }^{41}$, Lauren Gilbert ${ }^{41}$, Lee Graham ${ }^{41}$, Michelle Cronin ${ }^{41}$, Sara Kumziene-Summerhayes ${ }^{41}$, Sarah Taylor ${ }^{41}$, Sophie Jones ${ }^{41}$, Danielle C. Groves ${ }^{65}$, Peijun Zhang ${ }^{65}$, Marta Gallis ${ }^{65}$ \& Stavroula F. Louka ${ }^{65}$

\section{Samples \& logistics and software \& analysis tools} Igor Starinskij ${ }^{64}$

Sequencing \& analysis and software \& analysis tools

Chris Jackson ${ }^{15}$, Marina Gourtovaia ${ }^{44}$, Gerry Tonkin-Hill ${ }^{44}$, Kevin Lewis ${ }^{44}$,

Jaime M. Tovar-Corona ${ }^{44}$, Keith James ${ }^{44}$, Laura Baxter ${ }^{77}$, Mohammad T. Alam ${ }^{77}$,

Richard J. Orton ${ }^{64}$, Joseph Hughes ${ }^{64}$, Sreenu Vattipally ${ }^{64}$, Manon Ragonnet-Cronin ${ }^{79}$,

Fabricia F. Nascimento ${ }^{79}$, David Jorgensen ${ }^{79}$, Olivia Boyd ${ }^{79}$, Lily Geidelberg ${ }^{79}$. Alex E. Zarebski ${ }^{80}$ Jayna Raghwani ${ }^{80}$, Moritz U. G. Kraemer ${ }^{80}$, Joel Southgate ${ }^{26,41}$, Benjamin B. Lindsey ${ }^{65}$ \& Timothy M. Freeman ${ }^{65}$ 


\section{Software \& analysis tools and visualization}

Jon-Paul Keatley ${ }^{44}$, Joshua B. Singer ${ }^{64}$, Leonardo de Oliveira Martins ${ }^{71}$, Corin A. Yeats ${ }^{61}$ Khalil Abudahab ${ }^{61}$, Ben E. W. Taylor ${ }^{61}$ \& Mirko Menegazzo ${ }^{61}$

\section{Leadership \& supervision}

John Danesh ${ }^{44}$, Wendy Hogsden ${ }^{95}$, Sahar Eldirdiri ${ }^{101}$, Anita Kenyon ${ }^{101}$, Jenifer Mason ${ }^{119}$ Trevor I. Robinson ${ }^{119}$, Alison Holmes ${ }^{103,120}$, James Price ${ }^{103,120}$, John A. Hartley ${ }^{85}$, Tanya Curran ${ }^{53}$, Alison E. Mather ${ }^{71}$, Giri Shankar ${ }^{41}$, Rachel Jones ${ }^{41}$, Robin Howe ${ }^{41} \&$ Sian Morgan ${ }^{92}$

\section{Metadata curation}

Elizabeth Wastenge ${ }^{68}$, Michael R. Chapman ${ }^{2,44,121}$, Siddharth Mookerjee ${ }^{103,120}$, Rachael Stanley ${ }^{97}$, Wendy Smith ${ }^{111}$, Timothy Peto ${ }^{75}$, David Eyre ${ }^{75}$, Derrick Crook ${ }^{75}$, Gabrielle Vernet ${ }^{122}$, Christine Kitchen ${ }^{26}$, Huw Gulliver ${ }^{26}$, lan Merrick ${ }^{26}$, Martyn Guest ${ }^{26}$, Robert Munn ${ }^{26}$, Declan T. Bradley ${ }^{54,123}$ \& Tim Wyatt ${ }^{123}$

\section{Project administration}

Charlotte Beaver ${ }^{44}$, Luke Foulser ${ }^{44}$, Sophie Palmer ${ }^{2}$, Carol M. Churcher ${ }^{2}$, Ellena Brooks ${ }^{2}$ Kim S. Smith ${ }^{2}$, Katerina Galai ${ }^{2}$, Georgina M. McManus ${ }^{2}$, Frances Bolt ${ }^{103,120}$, Francesc Coll ${ }^{124}$ Lizzie Meadows ${ }^{71}$, Stephen W. Attwood ${ }^{80}$, Alisha Davies ${ }^{41}$, Elen De Lacy ${ }^{41}$, Fatima Downing ${ }^{41}$, Sue Edwards ${ }^{41}$, Garry P. Scarlett ${ }^{88}$, Sarah Jeremiah ${ }^{49}$ \& Nikki Smith ${ }^{65}$

\section{Samples \& logistics}

Danielle Leek ${ }^{2}$, Sushmita Sridhar ${ }^{2,44}$, Sally Forrest ${ }^{2}$, Claire Cormie ${ }^{2}$, Harmeet K. Gill ${ }^{2}$, Joana Dias ${ }^{2}$, Ellen E. Higginson ${ }^{2}$, Mailis Maes ${ }^{2}$, Jamie Young ${ }^{2}$, Michelle Wantoch ${ }^{118}$, Dorota Jamrozy ${ }^{44}$, Stephanie Lo ${ }^{44}$, Minal Patel ${ }^{44}$, Verity Hill ${ }^{69}$, Claire M. Bewshea ${ }^{84}$, Sian Ellard ${ }^{84,98}$, Cressida Auckland ${ }^{98}$, Ian Harrison ${ }^{63}$, Chloe Bishop ${ }^{63}$, Vicki Chalker ${ }^{63}$, Alex Richter ${ }^{125}$, Andrew Beggs ${ }^{125}$, Angus Best ${ }^{93}$, Benita Percival ${ }^{93}$, Jeremy Mirza ${ }^{93}$, Oliver Megram ${ }^{93}$, Megan Mayhew ${ }^{93}$, Liam Crawford ${ }^{93}$, Fiona Ashcroft ${ }^{93}$, Emma Moles-Garcia $^{93}$, Nicola Cumley ${ }^{93}$, Richard Hopes ${ }^{63}$, Patawee Asamaphan ${ }^{64}$, Marc O. Niebel ${ }^{64}$, Rory N. Gunson ${ }^{126}$, Amanda Bradley ${ }^{127}$, Alasdair Maclean ${ }^{127}$, Guy Mollett ${ }^{127}$, Rachel Blacow ${ }^{127}$, Paul Bird ${ }^{102}$, Thomas Helmer ${ }^{102}$, Karlie Fallon ${ }^{102}$, Julian Tang ${ }^{102}$, Antony D. Hale ${ }^{128}$,

Louissa R. Macfarlane-Smith ${ }^{128}$, Katherine L. Harper ${ }^{128}$, Holli Carden ${ }^{128}$, Nicholas W. Machin ${ }^{63,129}$, Kathryn A. Jackson ${ }^{78}$, Shazaad S. Y. Ahmad ${ }^{63,29}$, Ryan P. George ${ }^{129}$, Lance Turtle ${ }^{78}$, Elaine O’Toole ${ }^{119}$, Joanne Watts ${ }^{119}$, Cassie Breen ${ }^{119}$, Angela Cowell ${ }^{119}$, Adela Alcolea-Medina ${ }^{130,13}$ Themoula Charalampous ${ }^{43,90}$, Amita Patel ${ }^{52}$, Lisa J. Levett ${ }^{132}$, Judith Heaney ${ }^{132}$, Aileen Rowan ${ }^{79}$ Graham P. Taylor $^{79}$, Divya Shah ${ }^{91}$, Laura Atkinson ${ }^{91}$, Jack C. D. Lee ${ }^{91}$, Adam P. Westhorpe ${ }^{85}$ Riaz Jannoo ${ }^{85}$, Helen L. Lowe ${ }^{85}$, Angeliki Karamani ${ }^{85}$, Leah Ensel ${ }^{85}$, Wendy Chatterton ${ }^{132}$, Monika Pusok ${ }^{132}$, Ashok Dadrah ${ }^{96}$, Amanda Symmonds ${ }^{96}$, Graciela Sluga ${ }^{133}$, Zoltan Molnar ${ }^{54}$ Paul Baker ${ }^{106}$, Stephen Bonner ${ }^{106}$, Sarah Essex ${ }^{106}$, Edward Barton ${ }^{107}$, Debra Padgett ${ }^{107}$ Garren Scott ${ }^{107}$, Jane Greenaway ${ }^{108}$, Brendan A. I. Payne ${ }^{81}$, Shirelle Burton-Fanning ${ }^{81}$, Sheila Waugh ${ }^{81}$, Veena Raviprakash ${ }^{109}$, Nicola Sheriff ${ }^{109}$, Victoria Blakey ${ }^{109}$ Lesley-Anne Williams ${ }^{109}$, Jonathan Moore ${ }^{134}$, Susanne Stonehouse ${ }^{134}$, Louise Smith ${ }^{135}$ Rose K. Davidson ${ }^{72}$, Luke Bedford ${ }^{136}$, Lindsay Coupland ${ }^{97}$, Victoria Wright ${ }^{55}$ Joseph G. Chappell ${ }^{110}$, Theocharis Tsoleridis ${ }^{110}$, Jonathan Ball ${ }^{110}$, Manjinder Khakh ${ }^{111}$ Vicki M. Fleming ${ }^{111}$, Michelle M. Lister ${ }^{111}$, Hannah C. Howson-Wells ${ }^{111}$, Louise Berry ${ }^{111}$, Tim Boswell 111 , Amelia Joseph ${ }^{111}$, Iona Willingham ${ }^{111}$, Nichola Duckworth ${ }^{112}$, Sarah Walsh ${ }^{112}$, Emma Wise ${ }^{113,114}$, Nathan Moore ${ }^{113,114}$, Matilde Mori ${ }^{11,114,137}$, Nick Cortes ${ }^{113,114}$, Stephen Kidd ${ }^{113,114}$ Rebecca Williams $^{122}$, Laura Gifford ${ }^{41}$, Kelly Bicknell ${ }^{66}$, Sarah Wyllie ${ }^{66}$, Allyson Lloyd $^{66}$, Robert Impey ${ }^{66}$, Cassandra S. Malone ${ }^{76}$, Benjamin J. Cogger ${ }^{76}$, Nick Levene ${ }^{138}$, Lynn Monaghan ${ }^{138}$, Alexander J. Keeley ${ }^{65}$, David G. Partridge ${ }^{65,139}$, Mohammad Raza ${ }^{65,139}$ Cariad Evans ${ }^{65,139}$ \& Kate Johnson ${ }^{65,139}$

\section{Sequencing \& analysis}

Emma Betteridge ${ }^{44}$, Ben W. Farr ${ }^{44}$, Scott Goodwin ${ }^{44}$, Michael A. Quail ${ }^{44}$, Carol Scott ${ }^{44}$ Lesley Shirley ${ }^{44}$, Scott A. J. Thurston ${ }^{44}$, Diana Rajan ${ }^{44}$, Iraad F. Bronner ${ }^{44}$, Louise Aigrain ${ }^{44}$ Nicholas M. Redshaw ${ }^{44}$, Stefanie V. Lensing ${ }^{44}$, Shane McCarthy ${ }^{44}$, Alex Makunin ${ }^{44}$, Carlos E. Balcazar ${ }^{69}$, Michael D. Gallagher ${ }^{69}$, Kathleen A. Williamson ${ }^{69}$, Thomas D. Stanton ${ }^{69}$ Michelle L. Michelsen ${ }^{84}$, Joanna Warwick-Dugdale ${ }^{84}$, Robin Manley ${ }^{84}$, Audrey Farbos ${ }^{84}$ James W. Harrison ${ }^{84}$, Christine M. Sambles ${ }^{84}$, David J. Studholme ${ }^{84}$, Angie Lackenby ${ }^{63}$, Tamyo Mbisa ${ }^{63}$, Steven Platt ${ }^{63}$, Shahjahan Miah ${ }^{63}$, David Bibby ${ }^{63}$, Carmen Manso ${ }^{63}$, Jonathan Hubb ${ }^{63}$, Gavin Dabrera ${ }^{63}$, Mary Ramsay ${ }^{63}$, Daniel Bradshaw ${ }^{63}$, Ulf Schaefer ${ }^{63}$, Natalie Groves ${ }^{63}$, Eileen Gallagher ${ }^{63}$, David Lee ${ }^{63}$, David Williams ${ }^{63}$, Nicholas Ellaby ${ }^{63}$, Hassan Hartman ${ }^{63}$, Nikos Manesis ${ }^{63}$, Vineet Patel ${ }^{63}$, Juan Ledesma ${ }^{63}$, Katherine A. Twohig ${ }^{63}$, Elias Allara ${ }^{2,63}$, Clare Pearson ${ }^{2,63}$, Jeffrey K. J. Cheng ${ }^{7}$, Hannah E. Bridgewater ${ }^{7}$, Lucy R. Frost ${ }^{7}$ Grace Taylor-Joyce ${ }^{77}$, Paul E. Brown ${ }^{77}$, Lily Tong ${ }^{64}$, Alice Broos ${ }^{64}$, Daniel Mair ${ }^{64}$, Jenna Nichols ${ }^{64}$ Stephen N. Carmichael ${ }^{64}$, Katherine L. Smollett ${ }^{140}$, Kyriaki Nomikou ${ }^{64}$, Elihu Aranday-Cortes ${ }^{64}$, Natasha Johnson ${ }^{64}$, Seema Nickbakhsh ${ }^{64,73}$, Edith E. Vamos ${ }^{78}$, Margaret Hughes ${ }^{78}$,

Lucille Rainbow ${ }^{78}$, Richard Eccles ${ }^{78}$, Charlotte Nelson ${ }^{78}$, Mark Whitehead ${ }^{78}$, Richard Gregory ${ }^{78}$ Matthew Gemmell ${ }^{78}$, Claudia Wierzbicki ${ }^{78}$, Hermione J. Webster ${ }^{78}$, Chloe L. Fisher ${ }^{46}$, Adrian W. Signell ${ }^{141}$, Gilberto Betancor ${ }^{141}$, Harry D. Wilson ${ }^{141}$, Gaia Nebbia ${ }^{90}$, Flavia Flaviani ${ }^{142}$ Alberto C. Cerda ${ }^{131}$, Tammy V. Merrill ${ }^{131}$, Rebekah E. Wilson ${ }^{131}$, Marius Cotic ${ }^{85}$, Nadua Bayzid ${ }^{85}$ Thomas Thompson ${ }^{54}$, Erwan Acheson ${ }^{54}$, Steven Rushton ${ }^{143}$, Sarah O'Brien ${ }^{143}$, David J. Baker ${ }^{71}$, Steven Rudder ${ }^{71}$, Alp Aydin ${ }^{71}$, Fei Sang ${ }^{55}$, Johnny Debebe ${ }^{55}$, Sarah Francois ${ }^{80}$, Tetyana I. Vasylyeva ${ }^{80}{ }^{\circ}$, Marina Escalera Zamudio ${ }^{80}$, Bernardo Gutierrez ${ }^{80}$, Angela Marchbank ${ }^{26}$ Joshua Maksimovic ${ }^{92}$, Karla Spellman ${ }^{92}$, Kathryn McCluggage ${ }^{92}$, Mari Morgan ${ }^{41}$, Robert Beer ${ }^{92}$, Safiah Afifi ${ }^{92}$, Trudy Workman ${ }^{26}$, William Fuller ${ }^{26}$, Catherine Bresner ${ }^{26}$, Adrienn Angyal ${ }^{65}$, Luke R. Green ${ }^{65}$, Paul J. Parsons ${ }^{65}$, Rachel M. Tucker ${ }^{65}$, Rebecca Brown ${ }^{65}$ \& Max Whiteley ${ }^{65}$

\section{Software \& analysis tools}

James Bonfield ${ }^{44}$, Christoph Puethe ${ }^{44}$, Andrew Whitwham ${ }^{44}$, Jennifier Liddle ${ }^{44}$, Will Rowe ${ }^{33}$ Igor Siveroni ${ }^{79}$, Thanh Le-Viet ${ }^{71} \&$ Amy Gaskin ${ }^{41}$

\section{Wellcome Sanger Centre}

Irina Abnizova ${ }^{143}$, Louise Aigrain ${ }^{143}$ Alex Alderton ${ }^{143}$. Mozam Ali'143, Laura Allen ${ }^{143}$ Roberto Amato ${ }^{143}$, Ralph Anderson ${ }^{143}$, Cristina Ariani ${ }^{143}$, Siobhan Austin-Guest ${ }^{143}$, Sendu Bala ${ }^{143}$, Jeffrey Barrett ${ }^{143}$, Andrew Bassett ${ }^{143}$, Kristina Battleday ${ }^{143}$, James Beal ${ }^{143}$, Mathew Beale ${ }^{143}$, Charlotte Beaver ${ }^{143}$, Sam Bellany ${ }^{143}$, Tristram Bellerby ${ }^{143}$, Katie Bellis ${ }^{143}$, Duncan Berger ${ }^{143}$, Matt Berriman ${ }^{143}$, Emma Betteridge ${ }^{143}$, Paul Bevan ${ }^{143}$, Simon Binley ${ }^{143}$, Jason Bishop ${ }^{143}$, Kirsty Blackburn ${ }^{143}$, James Bonfield ${ }^{143}$, Nick Boughton ${ }^{143}$, Sam Bowker ${ }^{143}$, Timothy Brendler-Spaeth ${ }^{143}$, Iraad Bronner ${ }^{143}$, Tanya Brooklyn ${ }^{143}$, Sarah Kay Buddenborg ${ }^{143}$, Robert Bush ${ }^{143}$, Catarina Caetano ${ }^{143}$, Alex Cagan ${ }^{143}$, Nicola Carter ${ }^{143}$, Joanna Cartwright ${ }^{143}$, Tiago Carvalho Monteiro ${ }^{143}$, Liz Chapman ${ }^{143}$, Tracey-Jane Chillingworth ${ }^{143}$, Peter Clapham ${ }^{143}$ Richard Clark ${ }^{143}$, Adrian Clarke ${ }^{143}$, Catriona Clarke ${ }^{143}$, Daryl Cole ${ }^{143}$, Elizabeth Cook ${ }^{143}$, Maria Coppola ${ }^{143}$, Linda Cornell ${ }^{143}$, Clare Cornwell ${ }^{143}$, Craig Corton ${ }^{143}$, Abby Crackett ${ }^{143}$, Alison Cranage ${ }^{143}$, Harriet Craven ${ }^{143}$, Sarah Craw $^{143}$, Mark Crawford ${ }^{143}$, Tim Cutts ${ }^{143}$, Monika Dabrowska ${ }^{143}$, Matt Davies ${ }^{143}$, Robert Davies ${ }^{143}$, Joseph Dawson ${ }^{143}$, Callum Day ${ }^{143}$, Aiden Densem ${ }^{143}$, Thomas Dibling ${ }^{143}$, Cat Dockree ${ }^{143}$, David Dodd ${ }^{143}$, Sunil Dogga ${ }^{143}$, Matthew Dorman ${ }^{143}$, Gordon Dougan ${ }^{143}$, Martin Dougherty ${ }^{143}$, Alexander Dove ${ }^{143}$ Lucy Drummond ${ }^{143}$, Eleanor Drury ${ }^{143}$, Monika Dudek ${ }^{143}$, Jillian Durham ${ }^{143}$, Laura Durrant ${ }^{143}$, Elizabeth Easthope ${ }^{143}$, Sabine Eckert ${ }^{143}$, Pete Ellis ${ }^{143}$, Ben Farr ${ }^{143}$, Michael Fenton ${ }^{143}$, Marcella Ferrero ${ }^{143}$, Neil Flack ${ }^{143}$, Howerd Fordham ${ }^{143}$, Grace Forsythe ${ }^{143}$, Luke Foulser ${ }^{143}$, Matt Francis ${ }^{143}$, Audrey Fraser ${ }^{143}$, Adam Freeman ${ }^{143}$, Anastasia Galvin ${ }^{143}$, Maria Garcia-Casado ${ }^{143}$, Alex Gedny ${ }^{143}$, Sophia Girgis ${ }^{143}$, James Glover ${ }^{143}$, Sonia Goncalves ${ }^{143}$, Scott Goodwin ${ }^{143}$, Oliver Gould ${ }^{143}$, Marina Gourtovaia ${ }^{143}$. Andy Gray ${ }^{143}$, Emma Gray ${ }^{143}$, Coline Griffiths ${ }^{143}$ Yong $\mathrm{Gu}^{143}$, Florence Guerin ${ }^{143}$, Will Hamilton ${ }^{143}$, Hannah Hanks ${ }^{143}$, Ewan Harrison ${ }^{143}$, Alexandria Harrott ${ }^{143}$, Edward Harry ${ }^{143}$, Julia Harvison ${ }^{143}$, Paul Heath ${ }^{143}$, Anastasia Hernandez-Ko utoucheva $^{143}$, Rhiannon Hobbs $s^{143}$, Dave Holland ${ }^{143}$, Sarah Holmes ${ }^{143}$, Gary Hornett ${ }^{143}$, Nicholas Hough ${ }^{143}$, Liz Huckle ${ }^{143}$, Lena Hughes-Hallet ${ }^{143}$, Adam Hunter ${ }^{143}$, Stephen Inglis ${ }^{143}$, Sameena Iqbal ${ }^{143}$, Adam Jackson ${ }^{143}$, David Jackson ${ }^{143}$, Keith James ${ }^{143}$, Dorota Jamrozy ${ }^{143}$, Carlos Jimenez Verdejo ${ }^{143}$, Ian Johnston ${ }^{143}$, Matthew Jones ${ }^{143}$, Kalyan Kallepally ${ }^{143}$, Leanne Kane ${ }^{143}$, Keely Kay ${ }^{143}$, Sally Kay ${ }^{143}$, Jon Keatley ${ }^{143}$, Alan Keith ${ }^{143}$, Alison King ${ }^{143}$, Lucy Kitchin ${ }^{143}$, Matt Kleanthous ${ }^{143}$, Martina Klimekova ${ }^{143}$, Petra Korlevic ${ }^{143}$, Ksenia Krasheninnkova ${ }^{143}$, Dominic Kwiatkowski ${ }^{143}$, Greg Lane ${ }^{143}$, Cordelia Langford ${ }^{143}$, Adam Laverack ${ }^{143}$, Katharine Law ${ }^{143}$, Mara Lawniczak ${ }^{143}$, Stefanie Lensing ${ }^{143}$, Steven Leonard ${ }^{143}$, Laura Letchford ${ }^{143}$, Kevin Lewis ${ }^{143}$, Amanah Lewis-Wade ${ }^{143}$, Jennifer Liddle ${ }^{143}$, Quan Lin ${ }^{143}$, Sarah Lindsay ${ }^{143}$, Sally Linsdell ${ }^{143}$, Rich Livett ${ }^{143}$, Stephanie Lo ${ }^{143}$, Rhona Long ${ }^{143}$, Jamie Lovell ${ }^{143}$, Jon Lovell ${ }^{143}$, Catherine Ludden ${ }^{143}$, James Mack ${ }^{143}$. Mark Maddison ${ }^{143}$. Aleksei Makunin ${ }^{143}$, Irfan Mamun ${ }^{143}$, Jenny Mansfield ${ }^{143}$, Neil Marriott ${ }^{143}$, Matt Martin ${ }^{143}$, Inigo Martincorena ${ }^{143}$, Matthew Mayho ${ }^{143}$, Shane McCarthy ${ }^{143}$, Jo McClintock ${ }^{143}$, Samantha McGuigan ${ }^{143}$, Sandra McHugh ${ }^{143}$, Liz McMinn ${ }^{143}$, Carl Meadows ${ }^{143}$, Emily Mobley ${ }^{143}$, Robin Moll ${ }^{143}$, Maria Morra ${ }^{143}$, Leanne Morrow ${ }^{143}$, Kathryn Murie ${ }^{143}$, Sian Nash ${ }^{143}$, Claire Nathwani ${ }^{143}$ Plamena Naydenova ${ }^{143}$, Alexandra Neaverson ${ }^{143}$, Rachel Nelson ${ }^{143}$, Ed Nerou ${ }^{143}$, Jon Nicholson ${ }^{143}$, Tabea Nimz ${ }^{143}$, Guillaume G. Noell ${ }^{143}$, Sarah O'Meara ${ }^{143}$, Valeriu Ohan ${ }^{143}$, Karen Oliver ${ }^{143}$, Charles Olney ${ }^{143}$, Doug Ormond ${ }^{143}$, Agnes Oszlanczi ${ }^{143}$, Steve Palmer ${ }^{143}$, Yoke Fei Pang ${ }^{143}$, Barbora Pardubska ${ }^{143}$, Naomi Park ${ }^{143}$, Aaron Parmar ${ }^{143}$, Gaurang Patel ${ }^{143}$, Minal Patel ${ }^{143}$, Maggie Payne ${ }^{143}$, Sharon Peacock ${ }^{143}$, Arabella Petersen ${ }^{143}$, Deborah Plowman ${ }^{143}$, Tom Preston ${ }^{143}$, Liam Prestwood ${ }^{143}$, Christoph Puethe ${ }^{143}$, Michael Quail ${ }^{143}$, Diana Rajan ${ }^{143}$, Shavanthi Rajatileka ${ }^{143}$, Richard Rance ${ }^{143}$, Suzannah Rawlings ${ }^{143}$, Nicholas Redshaw ${ }^{143}$, Joe Reynolds ${ }^{143}$, Mark Reynolds ${ }^{143}$, Simon Rice ${ }^{143}$, Matt Richardson ${ }^{143}$, Connor Roberts ${ }^{143}$, Katrina Robinson ${ }^{143}$, Melanie Robinson ${ }^{143}$, David Robinson ${ }^{143}$, Hazel Rogers ${ }^{143}$, Eduardo Martin Rojo ${ }^{143}$, Daljit Roopra ${ }^{143}$, Mark Rose ${ }^{143}$, Luke Rudd ${ }^{143}$, Ramin Sadri ${ }^{143}$, Nicholas Salmon ${ }^{143}$, David Saul ${ }^{143}$, Frank Schwach ${ }^{143}$, Carol Scott ${ }^{143}$, Phil Seekings ${ }^{143}$, Lesley Shirley ${ }^{143}$, John Sillitoe ${ }^{143}$, Alison Simms ${ }^{143}$, Matt Sinnott ${ }^{143}$, Shanthi Sivadasan ${ }^{143}$ Bart Siwek ${ }^{143}$. Dale Sizer ${ }^{143}$, Kenneth Skeldon ${ }^{143}$, Jason Skelton ${ }^{143}$, Joanna Slater-Tunstill ${ }^{143}$ Lisa Sloper ${ }^{143}$, Nathalie Smerdon ${ }^{143}$, Chris Smith ${ }^{143}$, Christen Smith ${ }^{143}$, James Smith ${ }^{143}$, Katie Smith ${ }^{143}$, Michelle Smith ${ }^{143}$, Sean Smith ${ }^{143}$, Tina Smith ${ }^{143}$, Leighton Sneade ${ }^{143}$, Carmen Diaz Soria ${ }^{143}$, Catarina Sousa ${ }^{143}$, Emily Souster ${ }^{143}$, Andrew Sparkes ${ }^{143}$, Michael Spencer-Chapman ${ }^{143}$, Janet Squares ${ }^{143}$, Robert Stanley ${ }^{143}$, Claire Steed ${ }^{143}$, Tim Stickland ${ }^{143}$, Ian Still ${ }^{143}$, Mike Stratton $^{143}$, Michelle Strickland ${ }^{143}$, Allen Swann ${ }^{143}$ Agnieszka Swiatkowska ${ }^{143}$, Neil Sycamore ${ }^{143}$, Emma Swift $^{143}$, Edward Symons $^{143}$, Suzanne Szluha ${ }^{143}$, Emma Taluy ${ }^{143}$, Nunu Tao ${ }^{143}$, Katy Taylor ${ }^{143}$, Sam Taylor ${ }^{143}$, Stacey Thompson ${ }^{143}$, Mark Thompson ${ }^{143}$, Mark Thomson ${ }^{143}$, Nicholas Thomson ${ }^{143}$, Scott Thurston ${ }^{143}$, Gerry Tonkin-Hill ${ }^{143}$, Dee Toombs ${ }^{143}$, Benjamin Topping ${ }^{143}$, Jaime Tovar-Corona ${ }^{143}$, Daniel Ungureanu ${ }^{143}$, James Uphill ${ }^{143}$, Jana Urbanova ${ }^{143}$, Philip Jansen Van ${ }^{143}$, Valerie Vancollie ${ }^{143}$, Paul Voak ${ }^{143}$, Danielle Walker ${ }^{143}$, Matthew Walker ${ }^{143}$, Matt Waller ${ }^{143}$, Gary Ward ${ }^{143}$, Charlie Weatherhogg ${ }^{143}$, Niki Webb ${ }^{143}$, Danni Weldon ${ }^{143}$, Alan Wells ${ }^{143}$, Eloise Wells ${ }^{143}$, Luke Westwood ${ }^{143}$, Theo Whipp ${ }^{143}$, Thomas Whiteley ${ }^{143}$, Georgia Whitton ${ }^{143}$, Andrew Whitwham ${ }^{143}$, Sara Widaa ${ }^{143}$, Mia Williams ${ }^{143}$, Mark Wilson $^{143}$ \& Sean Wright ${ }^{143}$

${ }^{41}$ Public Health Wales NHS Trust, Cardiff, UK. ${ }^{42}$ Nuffield Department of Medicine, University of Oxford, Oxford, UK. ${ }^{43}$ Deparment of Virology, King's College London, London, UK. ${ }^{44}$ Department of Pathogen Genomics, Wellcome Sanger Institute, London, UK. ${ }^{45}$ Department of Medicine, University of Brighton, Brighton, UK. ${ }^{46} \mathrm{Genomics}$ Innovation Department, Guy's and St Thomas' NHS Foundation Trust, London, UK. ${ }^{47}$ Department of Infectious Diseases, Cambridge University Hospitals NHS Foundation Trust, Cambridge, UK. ${ }^{48}$ Department of Microbiology, Cambridge University Hospitals NHS Foundation Trust, Cambridge, UK. ${ }^{49}$ Department of Medicine, University Hospital Southampton NHS Foundation Trust, Southampton, UK. ${ }^{50}$ School of Medicine, Department of Medicine, University of Southampton, Southampton, UK. ${ }^{5}$ School of Health Sciences, Department of Medicine, University of Southampton, Southampton, UK. ${ }^{52}$ Department of Clinical Infection \& Diagnostics Research, St Thomas' Hospital and Kings College London, London, UK. ${ }^{53}$ Belfast Health \& Social Care Trust, Belfast, UK. ${ }^{54}$ Department of Medicine, Queen's University Belfast, Belfast, UK. ${ }^{55}$ Deep Seq Department, School of Life Sciences, Queens Medical Centre, University of Nottingham, Nottingham, UK. ${ }^{56}$ East Kent Hospitals University NHS Foundation Trust, Canterbury, UK. ${ }^{57}$ Department of Medicine, University of Kent, Canterbury, UK. ${ }^{58} \mathrm{Hub}$ for Biotechnology in the Built Environment, Northumbria University, Newcastle-Upon-Tyne, UK. ${ }^{59}$ Department of Medicine, Northumbria University, Newcastle-Upon-Tyne, UK. ${ }^{60} \mathrm{NU}$-OMICS, 
Northumbria University, Newcastle-upon-Tyne, UK. ${ }^{61}$ Centre for Genomic Pathogen Surveillance, University of Oxford, Oxford, UK. ${ }^{62}$ Clinical Microbiology and Public Health Laboratory, Public Health England, Cambridge, UK. ${ }^{63}$ Public Health England, London, UK. ${ }^{64} \mathrm{MRC}$-University of Glasgow Centre for Virus Research, Glasgow, UK. ${ }^{65}$ University of Sheffield, Sheffield, UK. ${ }^{66}$ Portsmouth Hospitals University NHS Trust, Portsmouth, UK. ${ }^{67}$ School of Pharmacy and Biomedical Sciences, University of Portsmouth (PORT), Portsmouth, UK. ${ }^{68} \mathrm{NHS}$ Lothian, Edinburgh, UK. ${ }^{69}$ University of Edinburgh, Edinburgh, UK. ${ }^{70}$ Department of Medicine, University of Oxford, Oxford, UK. ${ }^{71}$ Quadram Institute Bioscience, Norwich, UK. ${ }^{72}$ Department of Medicine, University of East Anglia, Norwich, UK. ${ }^{73}$ Public Health Scotland, Glasgow, UK. ${ }^{74} \mathrm{Heartlands} \mathrm{Hospital,} \mathrm{Birmingham,} \mathrm{UK.}{ }^{75}$ Department of Medicine, Oxford University Hospitals NHS Foundation Trust, Oxford, UK. ${ }^{76}$ Department of Medicine, Brighton and Sussex University Hospitals NHS Trust, Brighton, UK. ${ }^{77}$ Department of Medicine, University of Warwick, Warwick, UK. ${ }^{78}$ Department of Medicine, University of Liverpool, Liverpool, UK. ${ }^{79}$ Department of Medicine, Imperial College London, London, UK. ${ }^{80}$ Department of Zoology, University of Oxford, Oxford, UK. ${ }^{81}$ Newcastle Hospitals NHS Foundation Trust, Newcastle-upon-Tyne, UK. ${ }^{82}$ Division of Virology, Department of Pathology, University of Cambridge, Cambridge, UK. ${ }^{83}$ University Hospitals Coventry and Warwickshire, Coventry, UK. ${ }^{84}$ University of Exeter, Exeter, UK. ${ }^{85}$ University College London, London, UK. ${ }^{86}$ Wellcome Centre for Human Genetics, Nuffield Department of Medicine, University of Oxford, Oxford, UK. ${ }^{87}$ Bets Cadwaladr University Health Board, Betsi Cadwaladr, UK. ${ }^{88}$ School of Biological Sciences, University of Portsmouth (PORT), Portsmouth, UK. ${ }^{89}$ Warwick Medical School and Institute of Precision Diagnostics, Pathology, UHCW NHS Trust, Warwick, UK. ${ }^{90}$ Centre for Clinical Infection and Diagnostics Research, Department of Infectious Diseases, Guy's and St Thomas' NHS Foundation Trust, London, UK. ${ }^{91}$ Great Ormond Street Hospital for Children NHS Foundation Trust, London, UK. ${ }^{92}$ Cardiff and Vale University Health Board, Cardiff, UK. ${ }^{93}$ Turnkey Laboratory, University of Birmingham, Birmingham, UK.

${ }^{94}$ Gloucestershire Hospitals NHS Foundation Trust, Gloucester, UK. ${ }^{95}$ Microbiology Department, Wye Valley NHS Trust, Hereford, UK. ${ }^{96}$ Sandwell and West Birmingham NHS Trust, Birmingham, UK. ${ }^{97}$ Norfolk and Norwich University Hospital, Norwich, UK. ${ }^{98}$ Royal Devon and Exeter NHS Foundation Trust, Exeter, UK. ${ }^{99}$ Barking, Havering and Redbridge University Hospitals NHS Trust, Barking, UK. ${ }^{100}$ Queen Elizabeth Hospital, Birmingham, UK. ${ }^{101}$ Department of Microbiology, Kettering General Hospital, Kettering, UK. ${ }^{102}$ Clinical Microbiology, University Hospitals of Leicester NHS Trust, Leicester, UK. ${ }^{103}$ Imperial College Hospitals NHS Trust, London, UK. ${ }^{104}$ North West London Pathology, London, UK. ${ }^{105}$ Royal Free NHS Trust, London, UK. ${ }^{106}$ South Tees Hospitals NHS Foundation Trust, Newcastle-upon-Tyne, UK. ${ }^{107}$ North Cumbria Integrated Care NHS Foundation Trust, Carlisle, UK. ${ }^{108}$ North Tees and Hartlepool NHS Foundation Trust, Stockton-on-Tees, UK. ${ }^{109}$ County Durham and Darlington NHS Foundation Trust, Durham, UK. ${ }^{110}$ Virology, School of Life Sciences, Queens Medical Centre, University of Nottingham, Nottingham, UK. ${ }^{111} \mathrm{Clinical}$ Microbiology Department, Queens Medical Centre, Nottingham, UK. ${ }^{12}$ PathLinks, Northern Lincolnshire \& Goole NHS Foundation Trust, Scunthorpe, UK. ${ }^{113}$ Basingstoke Hospital, Basingstoke, UK. ${ }^{114}$ University of Surrey, Guildford, UK. ${ }^{115}$ Cambridge University Hospitals NHS Foundation Trust, Cambridge, UK. ${ }^{116}$ Swansea University, Swansea, UK. ${ }^{117}$ Ministry of Health, Colombo, Sri Lanka. ${ }^{118}$ Cambridge Stem Cell Institute, University of Cambridge, Cambridge, UK. ${ }^{119}$ Liverpool Clinical Laboratories, Liverpool, UK. ${ }^{120} \mathrm{NIHR}$ Health Protection Research Unit in HCAI and AMR, Imperial College London, London, UK. ${ }^{121}$ Health Data Research UK Cambridge, Cambridge, UK. ${ }^{122}$ Hampshire Hospitals NHS Foundation Trust, Winchester, UK. ${ }^{123}$ Public Health Agency, London, UK. ${ }^{124}$ Department of Infection Biology, Faculty of Infectious \& Tropical Diseases, London School of Hygiene \& Tropical Medicine, London, UK. ${ }^{125}$ University of Birmingham, Birmingham, UK. ${ }^{126}$ West of Scotland Specialist Virology Centre, NHS Greater Glasgow and Clyde, Glasgow, UK. ${ }^{127} \mathrm{NHS}$ Greater Glasgow and Clyde, Glasgow, UK. ${ }^{128}$ National Infection Service, PHE and Leeds Teaching Hospitals Trust, Leeds, UK. ${ }^{129}$ Manchester University NHS Foundation Trust, Manchester, UK. ${ }^{130}$ Guy's and St Thomas' Hospitals, London, UK. ${ }^{131}$ Viapath, Guy's and St Thomas' NHS Foundation Trust, and King's College Hospital NHS Foundation Trust, London, UK. ${ }^{132}$ Health Services Laboratories, London, UK. ${ }^{133}$ Maidstone and Tunbridge Wells NHS Trust, Maidstone, UK. ${ }^{134}$ Gateshead Health NHS Foundation Trust, Gateshead, UK. ${ }^{135}$ Norfolk County Council, Norwich, UK. ${ }^{136}$ East Suffolk and North Essex NHS Foundation Trust, Ipswich, UK. ${ }^{137}$ Department of Medicine, University of Southampton, Southampton, UK. ${ }^{138}$ Microbiology Department, Princess Alexandra Hospital, Harlow, UK. ${ }^{139}$ Sheffield Teaching Hospitals, Sheffield, UK. ${ }^{140}$ Institute of Biodiversity, Animal Health \& Comparative Medicine, Glasgow, UK. ${ }^{141}$ Department of Infectious Diseases, King's College London, London, UK. ${ }^{142}$ Guy's and St Thomas' BRC, London, UK. ${ }^{143}$ Well come Sanger Centre, Hinton, UK. 


\section{Methods}

\section{Data reporting}

No statistical methods were used to predetermine sample size. The experiments were not randomized and the investigators were not blinded to allocation during experiments and outcome assessment.

\section{Participant recruitment and ethics}

Participants who had received the first dose of the BNT162b2 vaccine and individuals with COVID-19 were consented into the COVID-19 cohort of the NIHR Bioresource. The study was approved by the East of England-Cambridge Central Research Ethics Committee (17/EE/0025).

\section{SARS-CoV-2 serology by multiplex particle-based flow cytometry}

Recombinant SARS-CoV-2 nucleocapsid, spike and RBD proteins were covalently coupled to distinct carboxylated bead sets (Luminex) to form a triplex and were analysed as previously described ${ }^{25}$. Specific binding was reported as the mean fluorescence intensity.

\section{Generation of spike mutants}

Amino acid substitutions were introduced into the D614G pCDNA_ SARS-CoV-2_S plasmid as previously described ${ }^{26}$ using the QuikChange Lightening Site-Directed Mutagenesis kit, following the manufacturer's instructions (Agilent Technologies). Sequences were checked by Sanger sequencing.

The plasmid encoding the B.1.1.7 or triple-mutant (spike(N501Y, E484K, K417N)) SARS-CoV-2 spike glycoprotein was used to produce SARS-CoV-2-MLVs based on overlap extension PCR as follows. In brief, a modification of the overlap extension PCR protocol ${ }^{27}$ was used to introduce the eight mutations of the B.1.1.7 lineage or the three mutations of the triple mutant (spike(N501Y, E484K, K417N)) in the SARS-CoV-2 spike gene. In a first step, nine DNA fragments with overlapping sequences were amplified by PCR from a plasmid (phCMV1, Genlantis) encoding the full-length SARS-CoV-2 spike gene (BetaCoV/Wuhan-Hu-1/2019; accession number, mn908947). The mutations $(\Delta H 69 / \Delta V 70, \Delta 144$, N501Y, A570D, D614G, P681H, S982A, T716I and D1118H or K417N, E484K and N501Y) were introduced by amplification with primers with a similar melting temperature. Deletion of the $\mathrm{C}$-terminal 21 amino acids was introduced to increase surface expression of the recombinant spike protein $^{28}$. Next, three contiguous overlapping fragments were fused by a first overlap PCR using the most external primers of each set, resulting in three larger fragments with overlapping sequences. A final overlap PCR was performed on the three large fragments using the most external primers to amplify the full-length spike gene and the flanking sequences including the restriction sites KpnI and NotI. This fragment was digested and cloned into the expression plasmid phCMV1. For all PCR reactions the Q5 Hot Start High Fidelity DNA polymerase (New England Biolabs) was used according to the manufacturer's instructions and adapting the elongation time to the size of the amplicon. After each PCR step, the amplified regions were separated on an agarose gel and purified using an Illustra GFX PCR DNA and Gel Band Purification Kit (Merck).

\section{Pseudotype virus preparation}

Viral vectors were prepared by transfection of HEK293T cells using the Fugene HD transfection reagent (Promega). HEK293T cells were transfected with a mixture of $11 \mu \mathrm{l}$ of Fugene HD, $1 \mu \mathrm{g}$ of pCDNAD19 spike-HA, $1 \mu \mathrm{g}$ of $\mathrm{p} 8.91 \mathrm{HIV}-1 \mathrm{Gag}-$ Pol expression vector ${ }^{29,30}$ and $1.5 \mu \mathrm{g}$ of pCSFLW (expressing the firefly luciferase reporter gene with the HIV-1 packaging signal) ${ }^{31}$. Viral supernatants were collected at $48 \mathrm{~h}$ and $72 \mathrm{~h}$ after transfection, filtered through a $0.45-\mu \mathrm{m}$ filter and stored at $-80{ }^{\circ} \mathrm{C}$. The $50 \%$ tissue culture infectious dose of the SARS-CoV- 2 pseudovirus was determined using the Steady-Glo Luciferase assay system (Promega).

\section{Serum and plasma pseudotype neutralization assay}

Spike pseudotype assays have been shown to have similar characteristics to neutralization tests using fully infectious wild-type SARS-CoV- $2^{32}$. Virus neutralization assays were performed on HEK293T cells that were transiently transfected with ACE2 and TMPRSS2 using a SARS-CoV-2 spike pseudotyped virus that expressed luciferase ${ }^{33}$. Pseudotyped virus was incubated with a serial dilution of heat-inactivated human serum samples or sera from individuals who were vaccinated in duplicate for $1 \mathrm{~h}$ at $37^{\circ} \mathrm{C}$. Virus-only and cell-only controls were also included. Then, freshly trypsinized HEK293T ACE2- and TMPRSS2-expressing cells were added to each well. After incubation for $48 \mathrm{~h}$ in a $5 \% \mathrm{CO}_{2}$ environment at $37^{\circ} \mathrm{C}$, luminescence was measured using the Steady-Glo or Bright-Glo Luciferase assay system (Promega). Neutralization was calculated relative to virus-only controls. Dilution curves are shown as the mean \pm s.e.m. neutralization. $\mathrm{ID}_{50}$ values were calculated in GraphPad Prism. The $\mathrm{ID}_{50}$ values within groups were summarized as the GMT and statistical comparisons between groups were made with Wilxocon ranked-sign tests. In addition, the effects of the mutations on the neutralizing effect of the sera were expressed as fold change in $\mathrm{ID}_{50}$ of the wild-type compared to mutant pseudotyped virus. Statistical difference in the mean fold change between groups was determined using a two-tailed Student's $t$-test.

\section{IFN $\gamma$ FluoroSpot assays}

Frozen peripheral blood mononuclear cells (PBMCs) were rapidly thawed, and the freezing medium was diluted into $10 \mathrm{ml}$ of TexMACS medium (Miltenyi Biotech), centrifuged and resuspended in $10 \mathrm{ml}$ of fresh medium with $10 \mathrm{U} \mathrm{ml}^{-1}$ DNase (Benzonase, Merck-Millipore via Sigma-Aldrich), PBMCs were incubated at $37^{\circ} \mathrm{C}$ for $1 \mathrm{~h}$, followed by centrifugation and resuspension in fresh medium supplemented with $5 \%$ human serum (Sigma-Aldrich) before being counted. PBMCs were stained with $2 \mu$ l of each antibody: anti-CD3-fluorescein isothiocyanate (FITC), clone UCHT1; anti-CD4-phycoerythrin (PE), clone RPA-T4; anti-CD8a-peridinin-chlorophyll protein-cyanine 5.5 (PerCP-Cy5.5), clone RPA-8a (all BioLegend, London, UK), LIVE/DEAD Fixable Far Red Dead Cell Stain Kit (Thermo Fisher Scientific). PBMC phenotyping was performed on the BD Accuri C6 flow cytometer. Data were analysed with FlowJo v.10 (Becton Dickinson). In brief, 1.5-2.5 $\times 10^{5}$ PBMCs were incubated in precoated Fluorospot plates (Human IFN $\gamma$ FLUOROSPOT (Mabtech)) in triplicate with peptide mixes specific to spike, nucleocapsid and membrane proteins of SARS-CoV-2 (final peptide concentration $1 \mu \mathrm{g} \mathrm{ml}^{-1}$ per peptide, Miltenyi Biotech) and an unstimulated and positive control mix (containing anti-CD3 (Mabtech), Staphylococcus Enterotoxin B, phytohaemagglutinin (all Sigma-Aldrich)) at $37^{\circ} \mathrm{C}$ in a humidified $\mathrm{CO}_{2}$ atmosphere for $48 \mathrm{~h}$. The cells and medium were decanted from the plate and the assay was developed following the manufacturer's instructions. Developed plates were read using an AID iSpot reader (Oxford Biosystems) and counted using AID EliSpot v.7 software (Autoimmun Diagnostika). All data were then corrected for background cytokine production and expressed as spot-forming units per million PBMCs or $\mathrm{CD}^{+}$T cells.

\section{Antibody discovery and recombinant expression}

Human monoclonal antibodies were isolated from plasma cells or memory B cells of donors who are immune to SARS-CoV or SARS-CoV-2 as previously described ${ }^{34-37}$. Recombinant antibodies were expressed in ExpiCHO cells at $37^{\circ} \mathrm{C}$ and $8 \% \mathrm{CO}_{2}$. Cells were transfected using ExpiFectamine. Transfected cells were supplemented 1 day after transfection with ExpiCHO Feed and ExpiFectamine CHO Enhancer. The cell culture supernatant was collected 8 days after transfection and filtered through a $0.2-\mu \mathrm{m}$ filter. Recombinant antibodies were affinity purified on an ÄKTA xpress FPLC device using 5-ml HiTrap MabSelect PrismA columns followed by buffer exchange to histidine buffer $(20 \mathrm{mM}$ histidine, $8 \%$ sucrose, pH 6) using HiPrep 26/10 desalting columns. 
Pseudovirus neutralization assay using monoclonal antibodies MLV-based SARS-CoV-2 S-glycoprotein-pseudotyped viruses were prepared as previously described ${ }^{35}$. HEK293T/17 cells were cotransfected with a plasmid encoding the wild-type, B.1.1.7 or triple-mutant (spike(N501Y, E484K, K417N)) SARS-CoV-2 spike glycoprotein, an MLV Gag-Pol packaging construct and the MLV transfer vector encoding a luciferase reporter using $X$-tremeGENE HP transfection reagent (Roche) according to the manufacturer's instructions. Cells were cultured for $72 \mathrm{~h}$ at $37^{\circ} \mathrm{C}$ with $5 \% \mathrm{CO}_{2}$ before collection of the supernatant. VeroE6 cells stably expressing human TMPRSS2 were cultured in Dulbecco's modified Eagle's medium (DMEM) containing $10 \%$ fetal bovine serum, $1 \%$ penicillin-streptomycin $\left(100 \mathrm{IU} \mathrm{ml}^{-1}\right.$ penicillin, $\left.100 \mathrm{\mu g} \mathrm{ml}^{-1}\right), 8 \mathrm{\mu g} \mathrm{ml}^{-1}$ puromycin and plated into $96-$-well plates for $16-24 \mathrm{~h}$. Pseudovirus with a serial dilution of monoclonal antibodies was incubated for $1 \mathrm{~h}$ at 37 ${ }^{\circ} \mathrm{C}$ and then added to the wells after washing twice with DMEM. After 2-3 h, DMEM containing 20\% fetal bovine serum and $2 \%$ penicillinstreptomycin was added to the cells. After 48-72 h of infection, Bio-Glo (Promega) was added to the cells and incubated in the dark for $15 \mathrm{~min}$ before the the luminescence was read using a Synergy $\mathrm{H} 1$ microplate reader (BioTek). Measurements were done in duplicate and relative luciferase units were converted to the percentage of neutralization and plotted with a nonlinear regression model to determine the $\mathrm{IC}_{50}$ values using GraphPad Prism software (v.9.0.0).

Antibody binding measurements using biolayer interferometry Monoclonal antibodies (Supplementary Table1) were diluted to $3 \mu \mathrm{g} \mathrm{ml}^{-1}$ in kinetic buffer (PBS supplemented with $0.01 \%$ BSA) and immobilized on Protein A Biosensors (FortéBio). Antibody-coated biosensors were incubated for $3 \mathrm{~min}$ with a solution containing $5 \mu \mathrm{g} \mathrm{ml}^{-1}$ of wild-type, N501Y or E484K SARS-CoV-2 RBD in kinetic buffer, followed by a 3-min dissociation step. Changes in the molecules bound to the biosensors caused a shift in the interference pattern that was recorded in real time using an Octet RED96 system (FortéBio). The binding response over time was used to calculate the area under the curve using GraphPad Prism software (v.9.0.0).

\section{Production of SARS-CoV-2 and B.1.1.7 RBDs and human ACE2}

The SARS-CoV-2 RBD (BEI NR-52422) construct was synthesized by GenScript into CMVR with an N-terminal mu-phosphatase signal peptide, a C-terminal octa-histidine tag (GHHHHHHHH) and an avi tag. The boundaries of the construct are ${ }_{328} \mathrm{RFPN}_{331}$ ( $\mathrm{N}$ terminus) and ${ }_{528} \mathrm{KKST}_{531}$ (C terminus) $)^{38}$. The B.1.1.7 RBD gene was synthesized by GenScript into pCMVR with the same boundaries and construct details with a mutation at N501Y. These plasmids were transiently transfected into Expi293F cells using Expi293F expression medium (Life Technologies) at $37^{\circ} \mathrm{C} 8 \% \mathrm{CO}_{2}$ while rotating at $150 \mathrm{rpm}$. The cultures were transfected using PEI cultivated for 5 days. Supernatants were clarified by centrifugation ( $10 \mathrm{~min}$ at $4,000 \mathrm{~g}$ ) before loading onto a nickel-NTA column (GE Healthcare). Purified protein was biotinylated overnight using BirA (Biotin ligase) before size-exclusion chromatography into PBS. Human ACE2-Fc (residues 1-615 with a C-terminal thrombin cleavage site and human Fctag) was synthesized by Twist. Clarified supernatants were affinity-purified using a Protein A column (GE Life Sciences) that was directly neutralized and buffer exchanged. The Fc tag was removed by thrombin cleavage in a reaction mixture containing $3 \mathrm{mg}$ of recombinant ACE2-Fc ectodomain and $10 \mu \mathrm{g}$ of thrombin in $20 \mathrm{mM}$ Tris- $\mathrm{HCl} \mathrm{pH} 8.0,150 \mathrm{mM} \mathrm{NaCland} 2.5 \mathrm{mM}$ $\mathrm{CaCl}_{2}$. The reaction mixture was incubated at $25^{\circ} \mathrm{C}$ overnight and reloaded on a Protein A column to remove uncleaved protein and the Fc tag. The cleaved protein was further purified by gel filtration using a Superdex 200 column 10/300 GL (GE Life Sciences) equilibrated in PBS.

Protein affinity measurements using biolayer interferometry Biotinylated RBDs (from wild-type spike, spike(N501Y) or spike(N501Y, $\mathrm{E} 484 \mathrm{~K}, \mathrm{~K} 417 \mathrm{~N})$ ) were immobilized at $5 \mathrm{ng} \mu \mathrm{l}$ in undiluted $10 \times$ Kinetics
Buffer (Pall) to SA sensors until a load level of $1.1 \mathrm{~nm}$. A dilution series of either monomeric ACE2 or Fab in undiluted kinetics buffer starting at 1,000 to $50 \mathrm{nM}$ was used for 300-600 s to determine protein-protein affinity. The data were baseline subtracted and the plots fitted using the Pall FortéBio/Sartorius analysis software (v.12.0). Data were plotted in Graphpad Prism (v.9.0.2).

\section{Phylogenetic analysis}

All complete and low-coverage-excluded SARS-CoV-2 sequences were downloaded from the GISAID database (http://gisaid.org/) ${ }^{39}$ on 11 February 2021. All sequences were realigned to the SARS-CoV-2 reference strain MN908947.3, using MAFFT v.7.475 with automatic flavour selection and the ---keeplength --addfragments options ${ }^{40}$. Sequences were then deduplicated. Major SARS-CoV-2 clade memberships were assigned to all sequences using the Nextclade server v.0.12 (https:// clades.nextstrain.org/).

Maximum likelihood phylogenetic trees were produced using the above curated dataset using IQ-TREE v.2.1.2.1. Evolutionary model selection for trees was inferred using ModelFinder ${ }^{10}$ and trees were estimated using the GTR $+\mathrm{F}+\mathrm{I}$ model with 1,000 ultrafast bootstrap replicates $^{42}$. All trees were visualized with Figtree v.1.4.4 (http://tree. bio.ed.ac.uk/software/figtree/) and manipulated and coloured with ggtree v.2.2.4. Phylogenies were rooted on the SARS-CoV-2 reference sequence (MN908947.3) and nodes arranged in descending order.

\section{Statistical analysis}

Linear regression was used to explore the association between the antibody response, $T$ cell response and serum neutralization in Stata 13. The Pearson correlation coefficient was reported.

\section{Neutralization data analysis}

Neutralization was calculated relative to virus-only controls. Dilution curves were presented as a mean \pm s.e.m. neutralization. $\mathrm{IC}_{50}$ values were calculated in GraphPad Prism. The ID $_{50}$ values within groups were summarized as a GMT and statistical comparisons between groups were made using Wilxocon ranked-sign tests. In addition, the effects of the mutations on the neutralizing effect of the sera were expressed as the fold change in $I_{50}$ of the wild-type compared to the mutant pseudotyped virus. Statistical difference in the mean fold change between groups was determined using a two-tailed Student's t-test.

\section{IFN $\gamma$ FluoroSpot assay data analysis}

The association between the spike-associated $\mathrm{T}$ cell response, spike-specific antibody response and serum neutralization was determined using linear regression. The Pearson correlation coefficients between these variables were determined using Stata 13.

\section{Reporting summary}

Further information on research design is available in the Nature Research Reporting Summary linked to this paper.

\section{Data availability}

The neutralization and biolayer interferometry data shown in Fig. 4 and Extended Data Figs. 6-8 can be found in the Source Data for Fig. 4. All sequences are publicly available and were downloaded from http:// gisaid.org. Deduplicated and subsampled data are freely available at https://github.com/StevenKemp/sequence_files/blob/main/vaccinepaper/with_background_subsampled_deduped_aligned_UKonly_484_vui. fasta.gz. Other data are available from the corresponding authors on request. Source data are provided with this paper.

25. Xiong, X. et al. A thermostable, closed SARS-CoV-2 spike protein trimer. Nat. Struct. Mol. Biol. 27, 934-941 (2020).

26. Gregson, J. et al. Human immunodeficiency virus-1 viral load is elevated in individuals with reverse-transcriptase mutation $\mathrm{M} 184 \mathrm{~V} / \mathrm{I}$ during virological failure of first-line 


\section{Article}

antiretroviral therapy and is associated with compensatory mutation L74I. J. Infect. Dis. 222, 1108-1116 (2019).

27. Forloni, M., Liu, A. Y. \& Wajapeyee, N. Creating insertions or deletions using overlap extension polymerase chain reaction (PCR) mutagenesis. Cold Spring Harb. Protoc. https://doi.org/10.1101/pdb.prot097758 (2018).

28. Case, J. B. et al. Neutralizing antibody and soluble ACE2 inhibition of a replication-competent VSV-SARS-CoV-2 and a clinical isolate of SARS-CoV-2. Cell Host Microbe 28, 475-485 (2020).

29. Naldini, L., Blömer, U., Gage, F. H., Trono, D. \& Verma, I. M. Efficient transfer, integration, and sustained long-term expression of the transgene in adult rat brains injected with a lentiviral vector. Proc. Natl Acad. Sci. USA 93, 11382-11388 (1996)

30. Gupta, R. K. et al. Full-length HIV-1 Gag determines protease inhibitor susceptibility within in-vitro assays. AIDS 24, 1651-1655 (2010)

31. Kemp, S. A. et al. SARS-CoV-2 evolution during treatment of chronic infection. Nature https://doi.org/10.1038/s41586-021-03291-y (2021).

32. Schmidt, F. et al. Measuring SARS-CoV-2 neutralizing antibody activity using pseudotyped and chimeric viruses. J. Exp. Med. 217, e2O2O1181 (2020).

33. Mlcochova, P. et al. Combined point of care nucleic acid and antibody testing for SARS-CoV-2 following emergence of D614G spike variant. Cell Rep. Med. 1, 100099 (2020).

34. Corti, D. et al. A neutralizing antibody selected from plasma cells that binds to group 1 and group 2 influenza A hemagglutinins. Science 333, 850-856 (2011).

35. Pinto, D. et al. Cross-neutralization of SARS-CoV-2 by a human monoclonal SARS-CoV antibody. Nature 583, 290-295 (2020).

36. Tortorici, M. A. et al. Ultrapotent human antibodies protect against SARS-CoV-2 challenge via multiple mechanisms. Science 370, 950-957 (2020).

37. Piccoli, L. et al. Mapping neutralizing and immunodominant sites on the SARS-CoV-2 spike receptor-binding domain by structure-guided high-resolution serology. Cell 183, 1024-1042 (2020)

38. Walls, A. C. et al. Elicitation of potent neutralizing antibody responses by designed protein nanoparticle vaccines for SARS-CoV-2. Cell 183, 1367-1382 (2020).

39. Shu, Y. \& McCauley, J. GISAID: global initiative on sharing all influenza data - from vision to reality. Euro Surveill. 22, 30494 (2017).

40. Katoh, K. \& Standley, D. M. MAFFT multiple sequence alignment software version 7: improvements in performance and usability. Mol. Biol. Evol. 30, 772-780 (2013).

41. Minh, B. Q. et al. IQ-TREE 2: new models and efficient methods for phylogenetic inference in the genomic era. Mol. Biol. Evol. 37, 1530-1534 (2020).

42. Minh, B. Q., Nguyen, M. A. \& von Haeseler, A. Ultrafast approximation for phylogenetic bootstrap. Mol. Biol. Evol. 30, 1188-1195 (2013).

Acknowledgements We thank the Cambridge University Hospitals NHS Trust Occupational Health Department; the NIHR Cambridge Clinical Research Facility and staff at CUH; E. Lim and G. Okecha; J. Voss for the gift of HeLa cells that stably express ACE2. R.K.G. is supported by a Wellcome Trust Senior Fellowship in Clinical Science (WT108082AIA). L.E.M. is supported by a Medical Research Council Career Development Award (MR/R008698/1). S.A.K. is supported by the Bill and Melinda Gates Foundation via PANGEA grant OPP1175094. D.A.C. is supported by a Wellcome Trust Clinical PhD Research Fellowship. K.G.C.S. is the recipient of a Wellcome Investigator Award (200871/Z/16/Z). This research was supported by the National Institute for Health Research (NIHR) Cambridge Biomedical Research Centre, the Cambridge Clinical Trials Unit (CCTU) and the NIHR BioResource. This study was supported by the National Institute of General Medical Sciences (R01GM120553 to D.V.), the National Institute of Allergy and Infectious Diseases (DP1Al158186 and HHSN272201700059C to D.V.), a Pew Biomedical Scholars Award (D.V.), an Investigators in the Pathogenesis of Infectious Disease Awards from the Burroughs Wellcome Fund (D.V.) and Fast Grants (D.V.). The views expressed are those of the authors and not necessarily those of the NIHR or the Department of Health and Social Care. I.A.T.M.F. is funded by a SANTHE award (DEL-15-006)

Author contributions D.C., R.K.G. and D.A.C. conceived the study. R.K.G., D.A.C., L.E.M., J. Bassi, M.W., L.C.-G., G.B.-M., R.D., B.G., N.K., A.E., M.S.P., D.V., L.P., A.D.M., J.R.B. and D.C. designed the study and experiments. B.M., D.A.C., N.T., R.P.D., I.A.T.M.F., A.C.W., L.C.-G., S.A.K. and G.B.-M performed experiments. R.K.G., D.A.C., B.M., R.D., I.A.T.M.F., A.C.W., L.E.M., J. Bassi, K.G.C.S. and D.V. interpreted data. A.D.M., and C.S.F. carried out pseudovirus neutralization assays. D.P. produced pseudoviruses. M.S.P., L.P., W.H., D.V. and D.C. designed the experiments. M.A.T., J. Bassi and S.J. expressed and purified the proteins. K.C., S.J. and E.C. sequenced and expressed antibodies. E.C. and K.C. performed mutagenesis to create mutant expression plasmids. A.C.W. and S.B. performed binding assays. A.R., A.F.P. and C.G. contributed to the recruitment of donors and collection of samples related to the isolation of monoclona antibodies. H.W.V., G.S., A.L., D.V., L.P., D.V. and D.C. analysed the data and prepared the manuscript with input from all authors.

Competing interests A.D.M., J. Bassi, D.P., C.S.F., S.B., K.C., N.S., E.C., G.S., S.J., A.L., H.W.V., M.S.P., L.P. and D.C. are employees of Vir Biotechnology and may hold shares in Vir Biotechnology. H.W.V. is a founder of PierianDx and Casma Therapeutics. Neither company provided funding for this work or is performing related work. D.V. is a consultant for Vir Biotechnology. The Veesler laboratory has received a sponsored research agreement from Vir Biotechnology. R.K.G. has received consulting fees from UMOVIS Lab, Gilead and ViiV. The ther authors declare no competing interests.

Additional information

Supplementary information The online version contains supplementary material available at https://doi.org/10.1038/s41586-021-03412-7.

Correspondence and requests for materials should be addressed to D.C. or R.K.G.

Peer review information Nature thanks the anonymous reviewers for their contribution to the peer review of this work. Peer reviewer reports are available.

Reprints and permissions information is available at http://www.nature.com/reprints. 


\section{a}

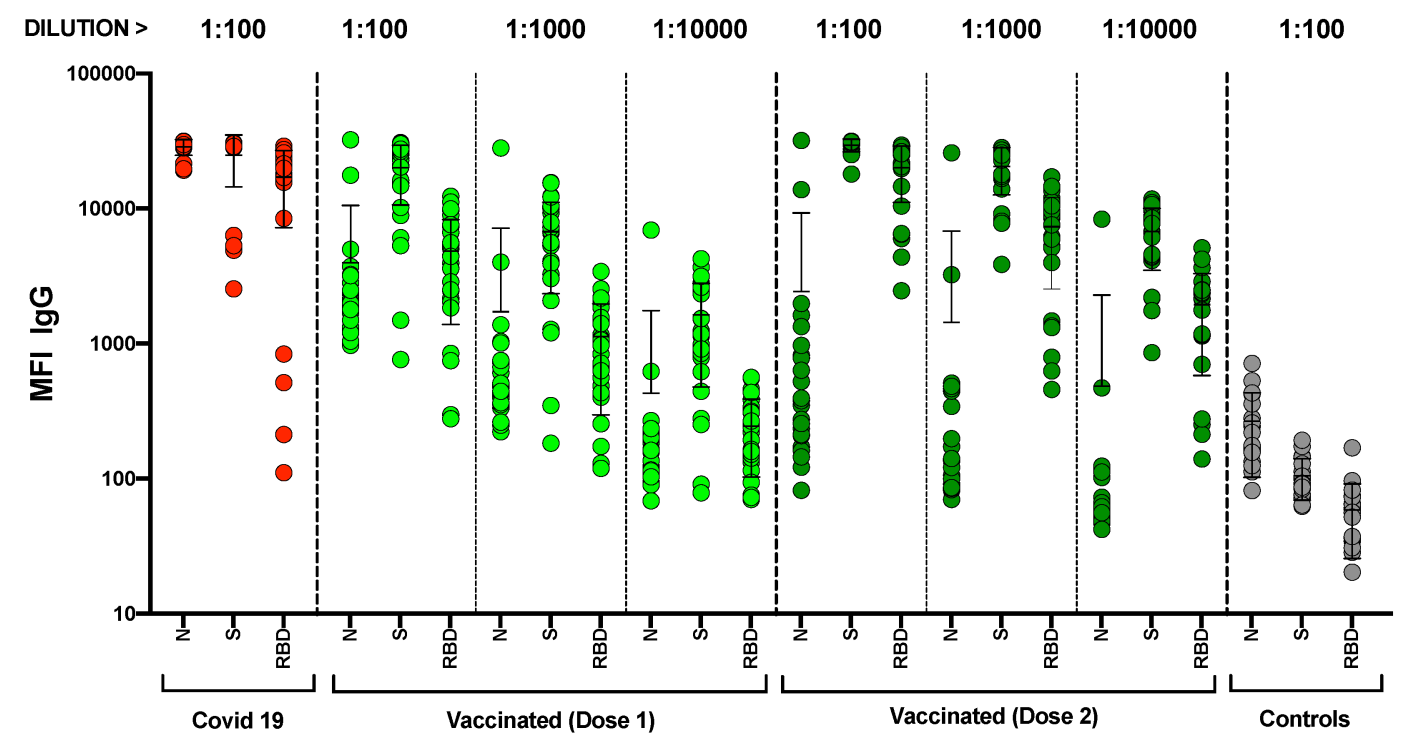

b

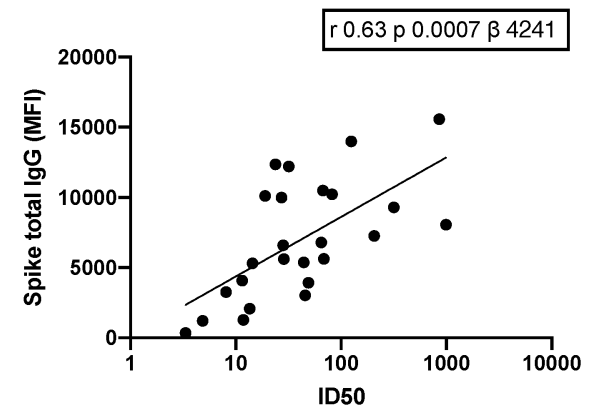

C

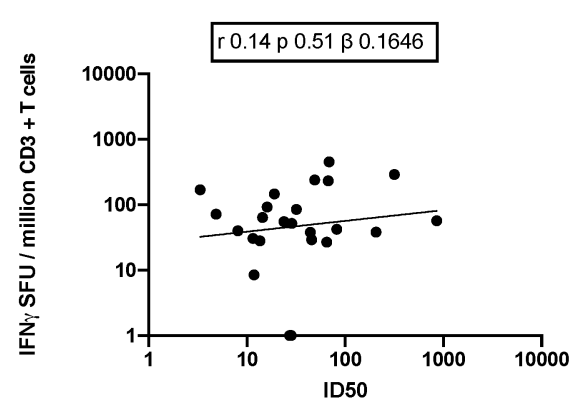

d

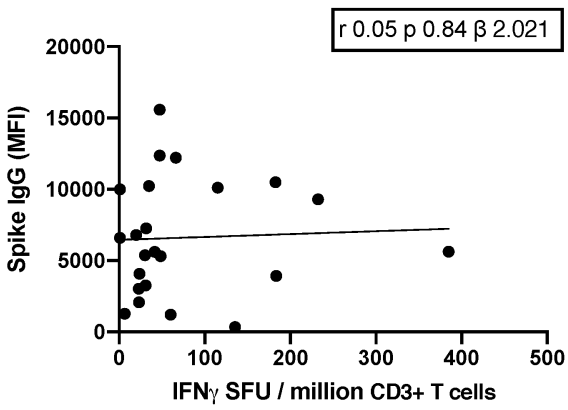

Extended Data Fig. 1 Immune responses three weeks after first dose of the BNT162b2 vaccine against SARS-CoV-2. a, Serum IgG responses against the $N$ protein, spike protein and the RBD of the spike protein of participants who received one vaccine dose (light green) or two vaccine doses (dark green), patients who had recovered from COVID-19 (red) and healthy control individuals (grey) were measured by a flow-cytometry-based Luminex assay. $n$ $=25$. MFI, mean fluorescence intensity. Data are GMT \pm s.d. (lines and error bars) of two technical repeats and individual values (cirlces).

b, Relationship between serum IgG responses, measured by flow cytometry, and serum neutralization $\mathrm{ID}_{50} . n=25$. c, Relationship between serum neutralization $\mathrm{ID}_{50}$ and $\mathrm{T}$ cell responses against SARS-CoV-2 by IFN $\gamma$ FluoroSpot. $n=24$. SFU, spot-forming units. d, Relationship between serum IgG responses and T cell responses. $n=23$. b-d, Simple linear regressions are shown with Pearson correlation (r), $P$ value $(p)$ and regression coefficient/slope $(\beta)$. 

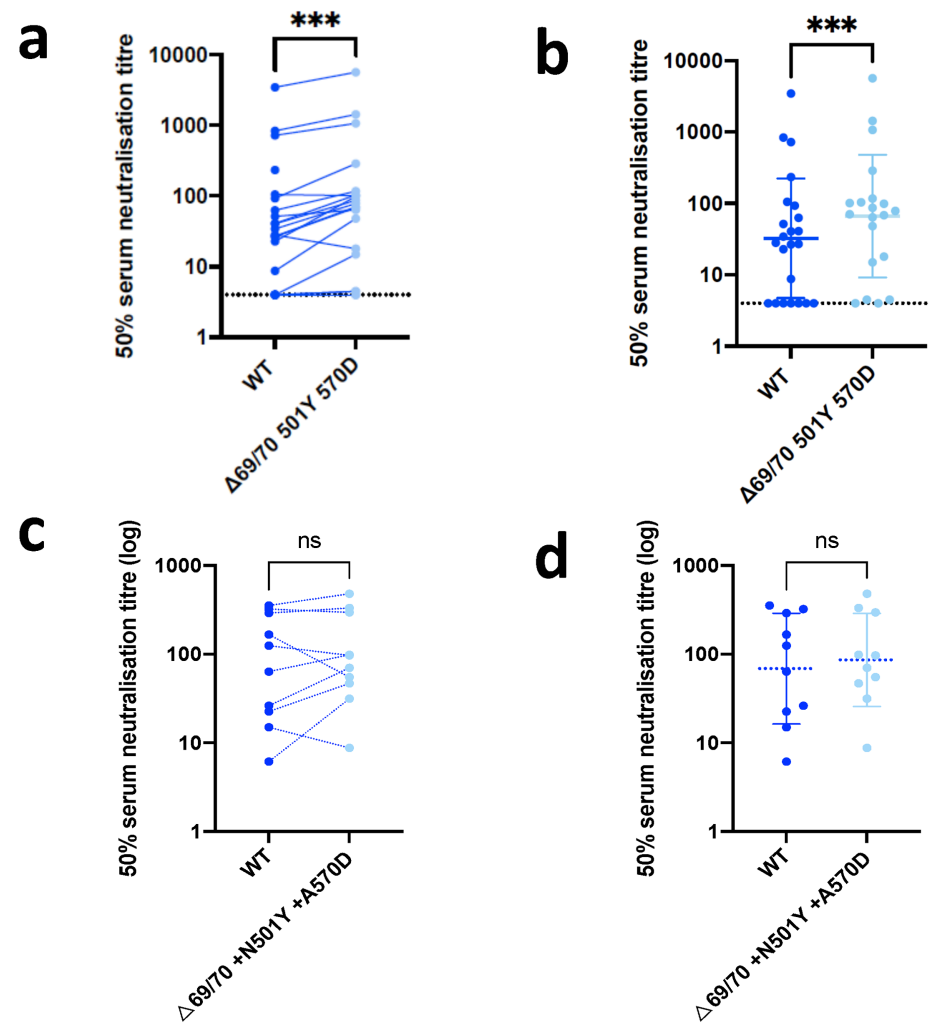

e
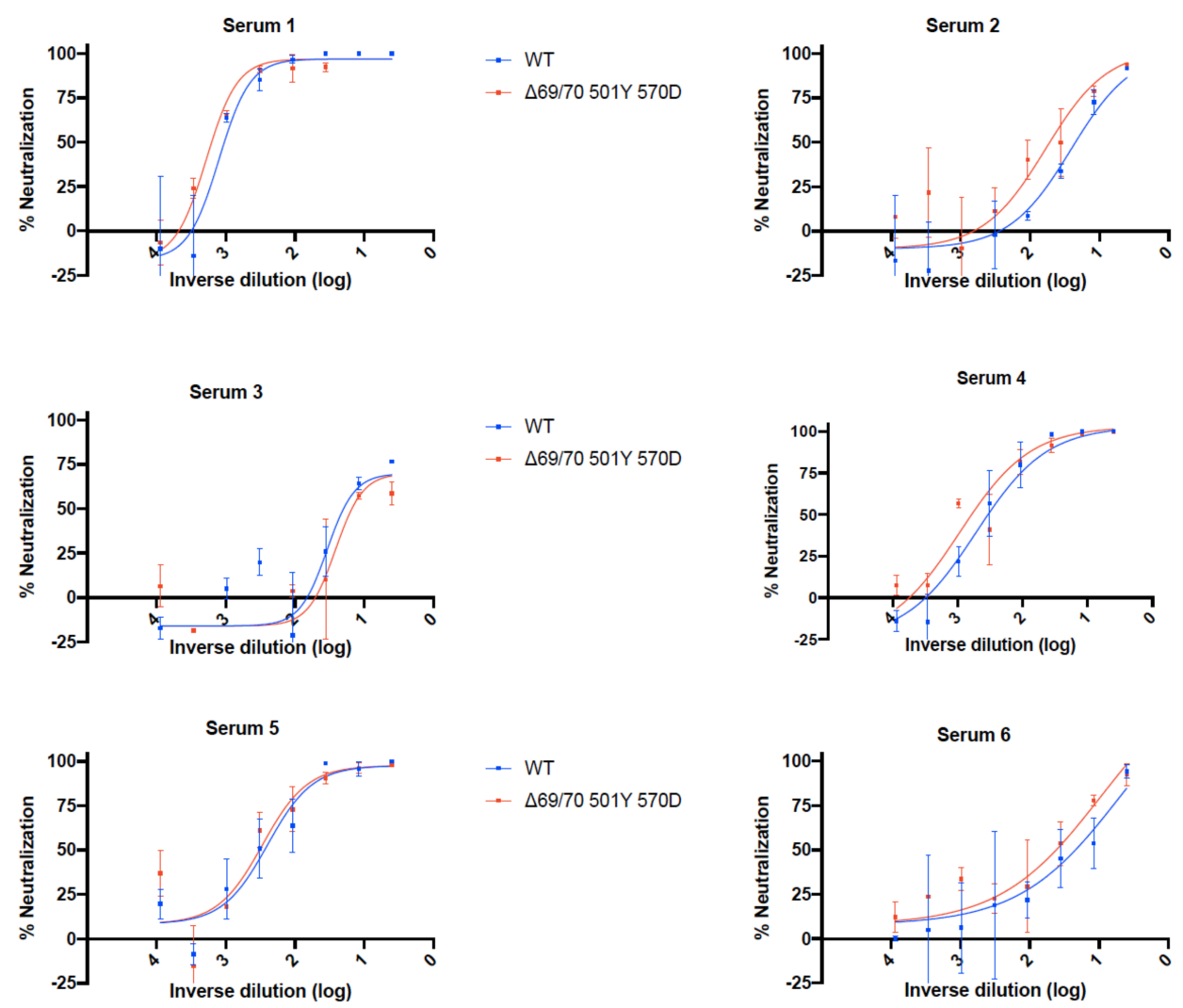

Extended Data Fig. 2 |See next page for caption. 
Extended Data Fig. 2 | Neutralization by the first dose of the BNT162b2 vaccine and convalescent sera against the wild-type and mutant (N501Y, A570D, $\Delta$ H69/AV70) SARS-CoV-2-pseudotyped viruses. a, b, Dilution of the vaccine sera for $50 \%$ neutralization against the wild-type and spike mutant (N501Y, A570D, $\Delta$ H69/AV70) viruses. b, Data are GMT \pm s.d. (lines and error bars) of two independent experiments with two technical repeats and individual values (circles). Two-tailed Wilcoxon matched-pairs signed-rank test with no adjustment for multiple comparisons; ${ }^{* * *} P<0.001$. c, d, Dilution of convalescent sera for $50 \%$ neutralization against the wild-type and spike

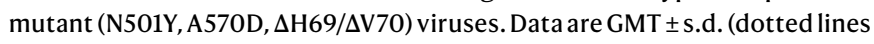
and error bars) of a representative experiment with two technical repeats and individual values (circles). Two-tailed Wilcoxon matched-pairs signed-rank test with no adjustment for multiple comparisons; ns, not significant. e, Representative curves of $\log _{10}$-transformed inverse dilutions of convalescent sera against the percentage of neutralization for the wild-type and spike mutant (N501Y, A570D, $\Delta \mathrm{H} 69 / \Delta \mathrm{V} 70$ ) viruses. In cases in which a curve is shifted to the right the virus is less sensitive to the neutralizing antibodies in the serum. Data are mean \pm s.e.m. of two technical replicates. Data are representative of two independent experiments. The cut-off for $50 \%$ neutralization was set to 4 (dotted lines in a, b). a, c, Data points of the same individual are connected by lines. 
ID 2 D1

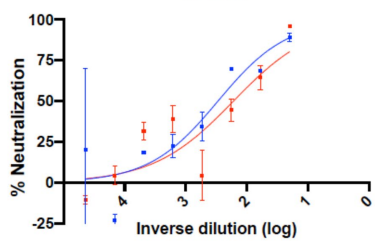

ID 15 D1

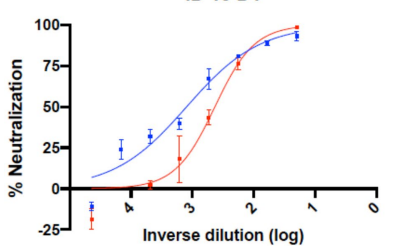

ID 24 D1

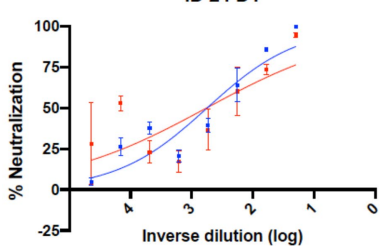

ID 3 D1

$\rightarrow W T$

$\rightarrow$ B.1.1.7
ID 9 D1

-WT

- B.1.1.7

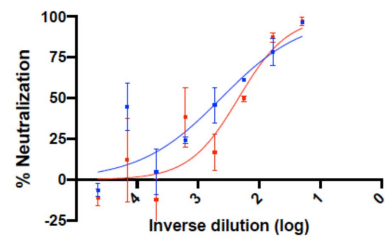

- WT

- B.1.1.7
ID 30 D1

- WT
- B.1.1.7

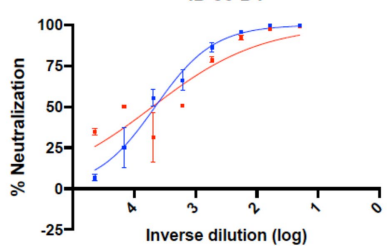

- WT

- B.1.1.7

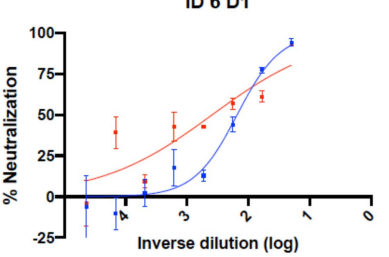

- WT

- B.1.1.7

$\rightarrow W T$

- B.1.1.7

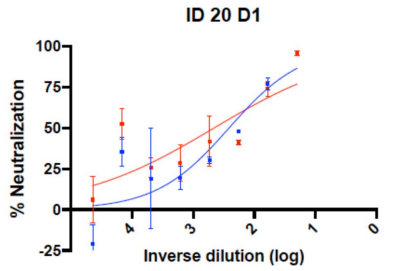

- WT

- B.1.1.7

Extended Data Fig. 3 | Representative neutralization curves of sera from individuals vaccinated with the BNT162b2 vaccine against pseudotyped virus bearing eight mutations in the spike protein that are present in the B.1.1.7 variant compared with the wild-type virus. All virus variants were in a spike(D614G) background. The $\log _{10}$-transformed inverse dilutions of the sera are shown against the percentage of neutralization. In cases in which a curve is shifted to the right the virus is less sensitive to the neutralizing antibodies in the serum. Data are for the first dose of vaccine (D1). Data are mean \pm s.e.m. representative of two independent experiments each with two technical replicates. 
Serum 3008

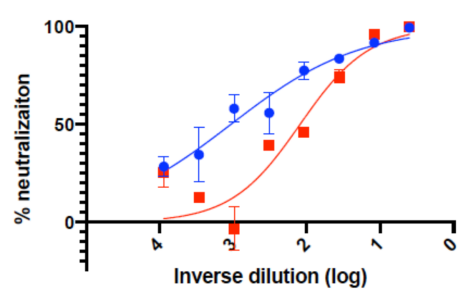

Serum 3015

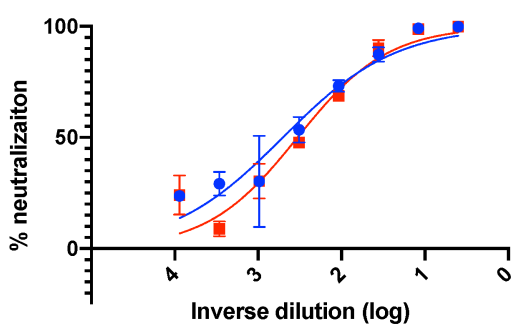

Serum 3037

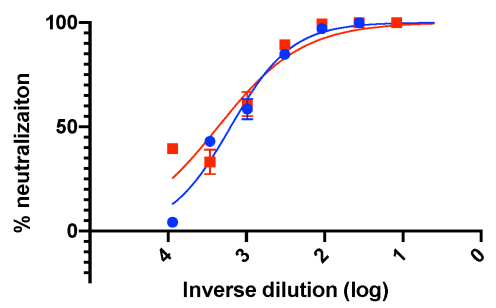

$\rightarrow W T$

- B.1.1.7 $\rightarrow$ WT

- B.1.1.7

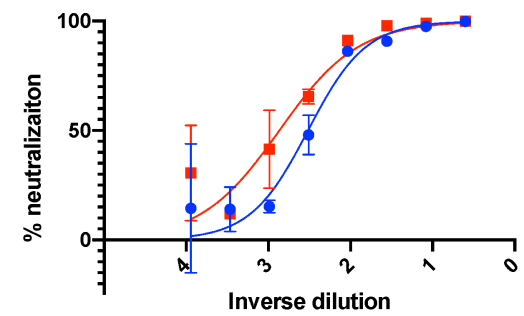

Serum 3047

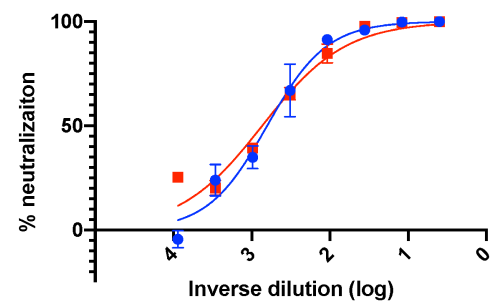

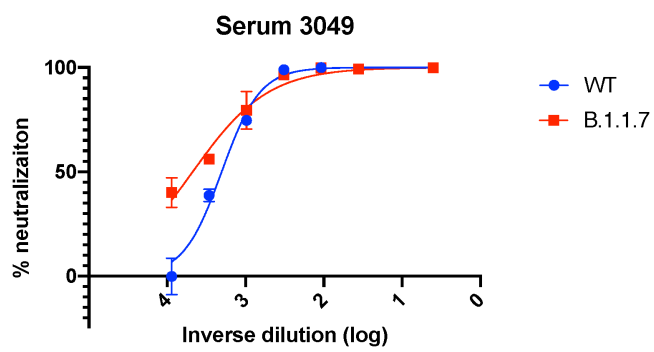

Extended Data Fig. 4 | Representative neutralization curves of convalescent sera against wild-type and B.1.1.7 spike-mutant SARS-CoV-2 pseudotyped viruses. The $\log _{10}$-transformed inverse dilutions of the sera are shown against the percentage of neutralization. In cases in which a curve is

shifted to the right the virus is less sensitive to the neutralizing antibodies in the serum. Data are mean \pm s.e.m. representative of two independent experiments each with two technical replicates. 
Article

Dose 1

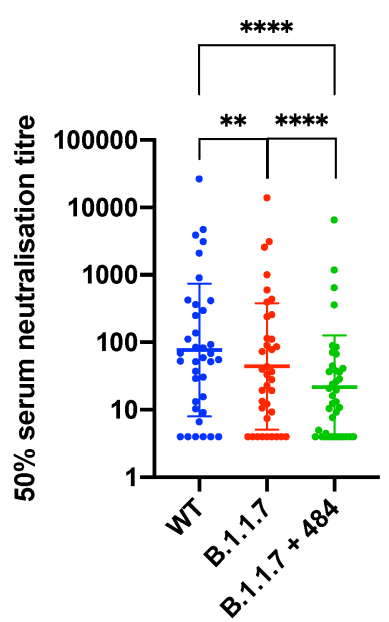

Extended Data Fig. 5 | Neutralization potency of $m$ RNA vaccine ser and convalescent vera (before SARS-CoV-2 B.1.1.7) against pseudotyped virus bearing spike mutations in the $B .1 .1 .7$ lineage with and without the $E 484 K$ substitution in the RBD. All virus variants were in a spike(D614G) background. Neutralization potency of the era from the first (left; $n=37)$ and the second (middle, $n=21$ ) vaccine dose and of convalescent plasma (CP) (right; $n=27$ )
Dose 2

$\mathrm{CP}$
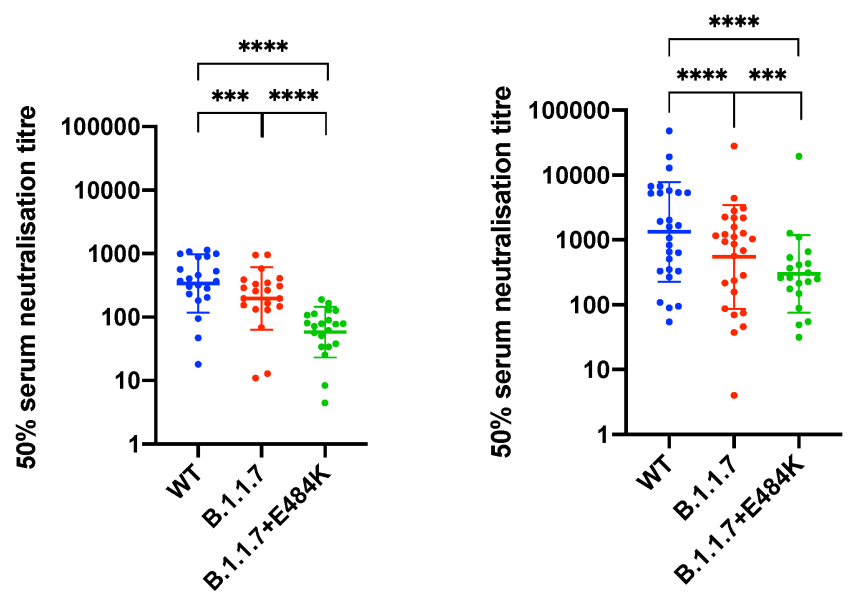

against wild-type SARS-CoV-2, the B.1.1.7 variant with spike(N501Y, A570D, $\Delta \mathrm{H} 69 / \Delta \mathrm{V} 70, \Delta 144, \mathrm{P} 681 \mathrm{H}, \mathrm{T} 716 \mathrm{I}, \mathrm{S} 982 \mathrm{~A}, \mathrm{D} 1118 \mathrm{H})$ and the B.1.1.7 variant with spike(N501Y, A570D, $\Delta$ H69/AV70, $\Delta 144$, P681H, T716I,S982A, D1118H) and the additional $\mathrm{E} 484 \mathrm{~K}$ substitution. Data are $\mathrm{GMT} \pm$ s.d. representative of two independent experiments each with two technical repeats. Wilcoxon matched-pairs signed-rank test; ${ }^{* *} P<0.01,{ }^{* * *} P<0.001,{ }^{* * * *} P<0.0001$ 
a
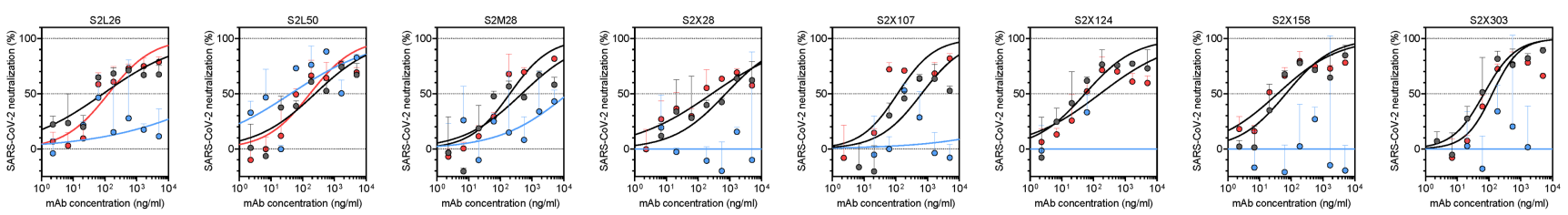

b
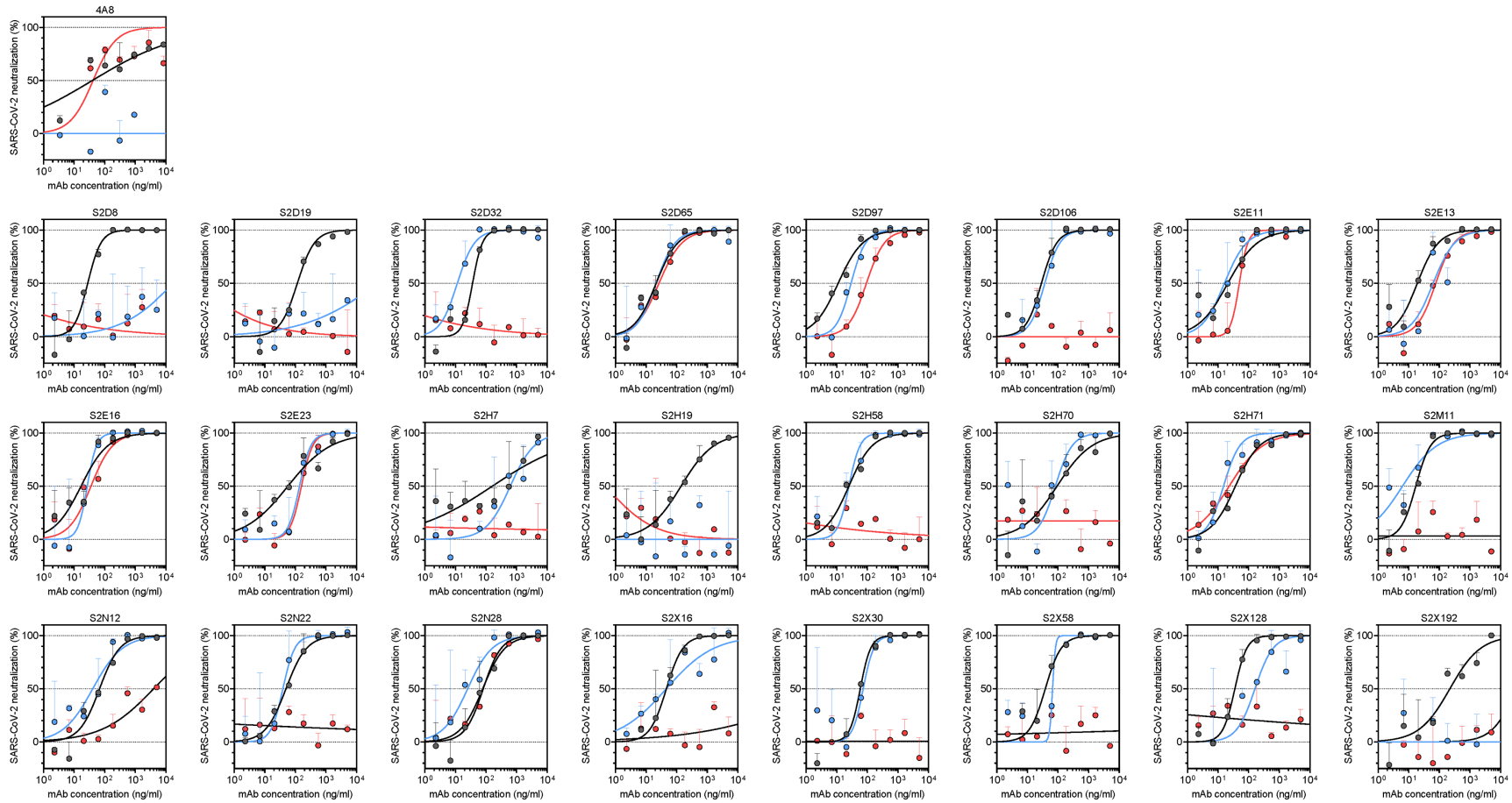

C
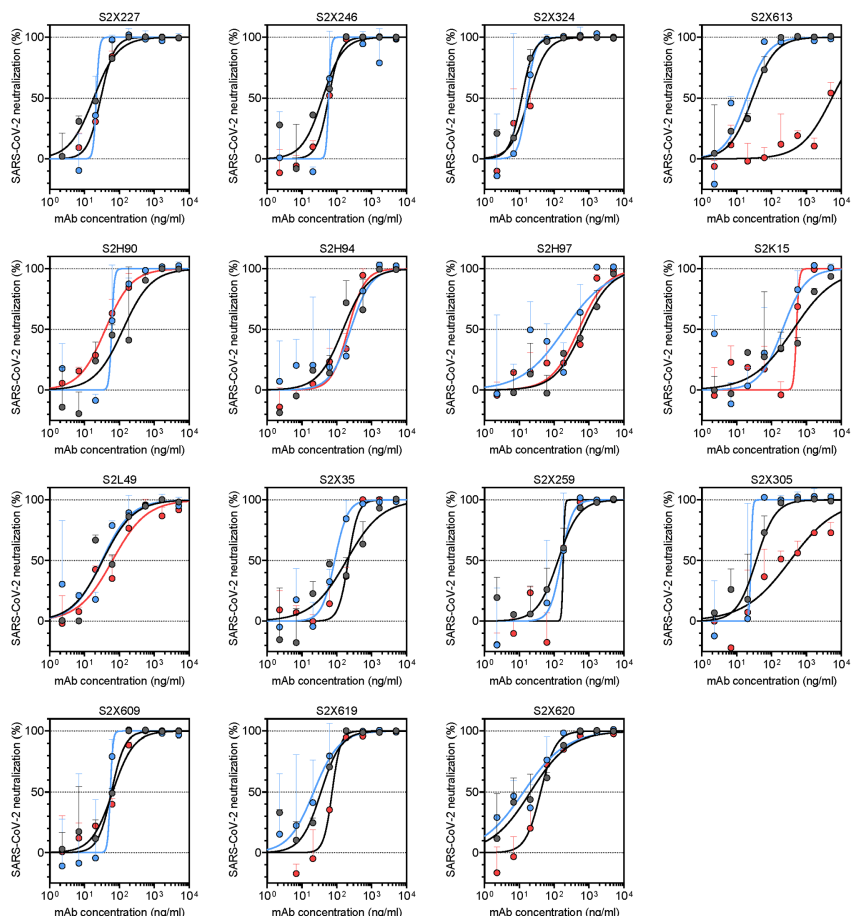

Extended Data Fig. 6 | Neutralization of the wild-type spike(D614G), B.1.1.7 spike and spike(N501Y, E484K, K417N) proteins of the SARS-CoV2-pseudotyped virus by a panel of 57 monoclonal antibodies.

a-c, Neutralization of the wild-type spike (black), B.1.1.7 spike (blue) and
spike(N501Y, E484K, K417N) (TM) (red) SARS-CoV-2-MLV by 9 NTD-targeting (a), 29 RBM-targeting (b) and 19 non-RBM-targeting (c) monoclonal antibodies. Data are mean \pm s.d. of two technical replicates from one representative experiment. 
a
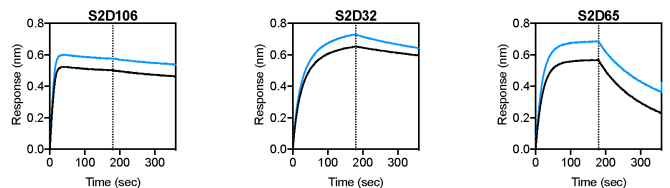

S2E23
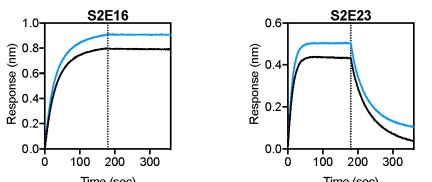

Time (sec)
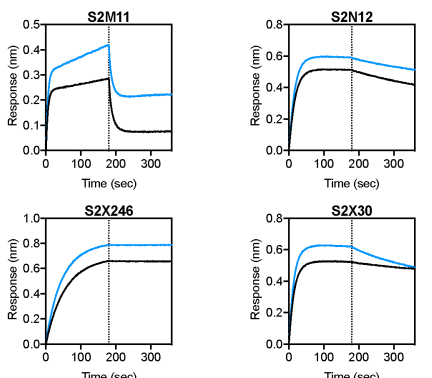

b
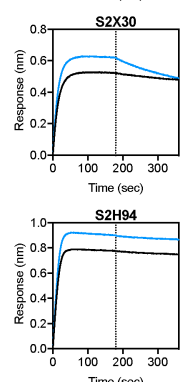

Time (sec)
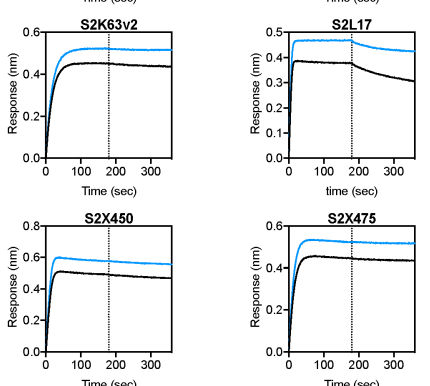

Time (sec)
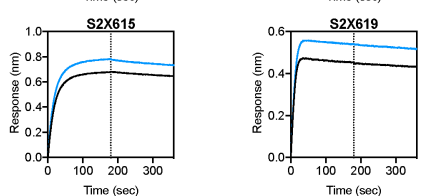
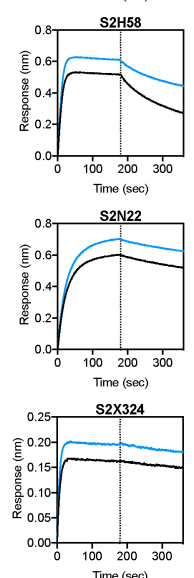

$\$ 2497$

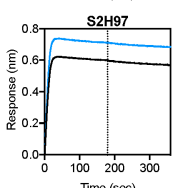

Time (sec)
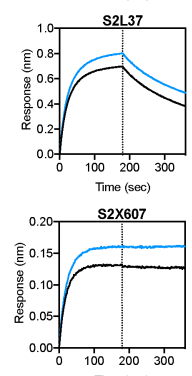

Time (sec)

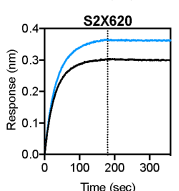

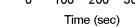

Extended Data Fig. 7 | Kinetics of binding to the RBD of wild-type and spike(N501Y) SARS-CoV-2 for 43 RBD-specific monoclonal antibodies. $\mathbf{a}-\mathbf{b}, \mathbf{a}, \mathbf{b}$, Binding to the RBD of wild-type (black) and spike(N501Y) (blue)
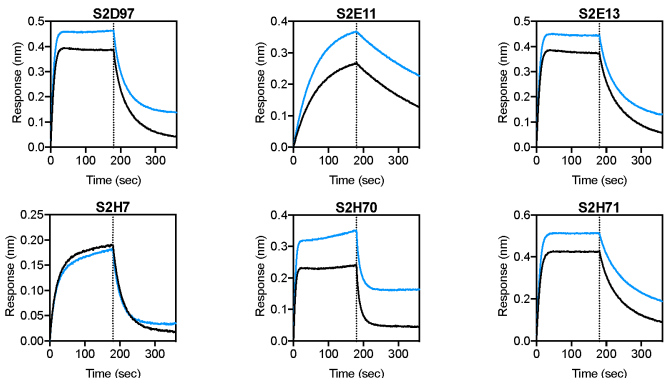

$s 2 H 70$
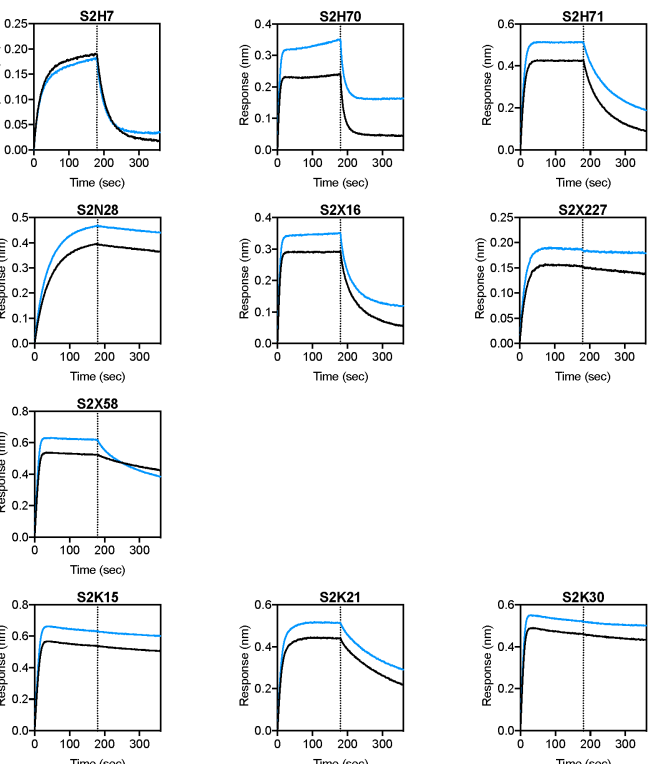

Time $($ sog
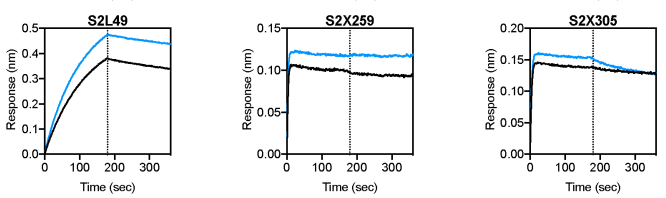

$\mathbf{5 2 \times 6 0 8}$
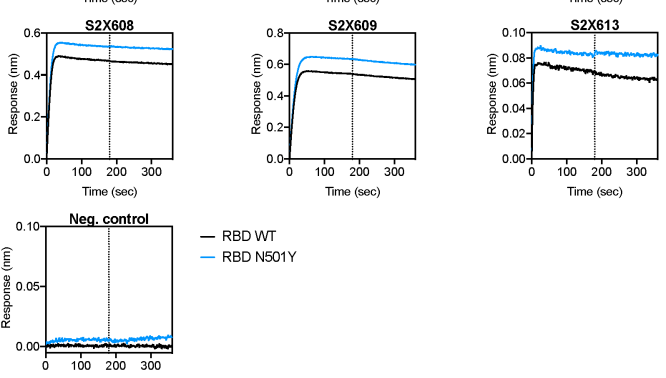

- RBD WT

- RBD N501Y

Time (sec)

SARS-CoV-2 by 22 RBM-targeting (a) and 21 non-RBM-targeting (b) monoclonal antibodies. An antibody of irrelevant specificity was included as a negative control. 
a
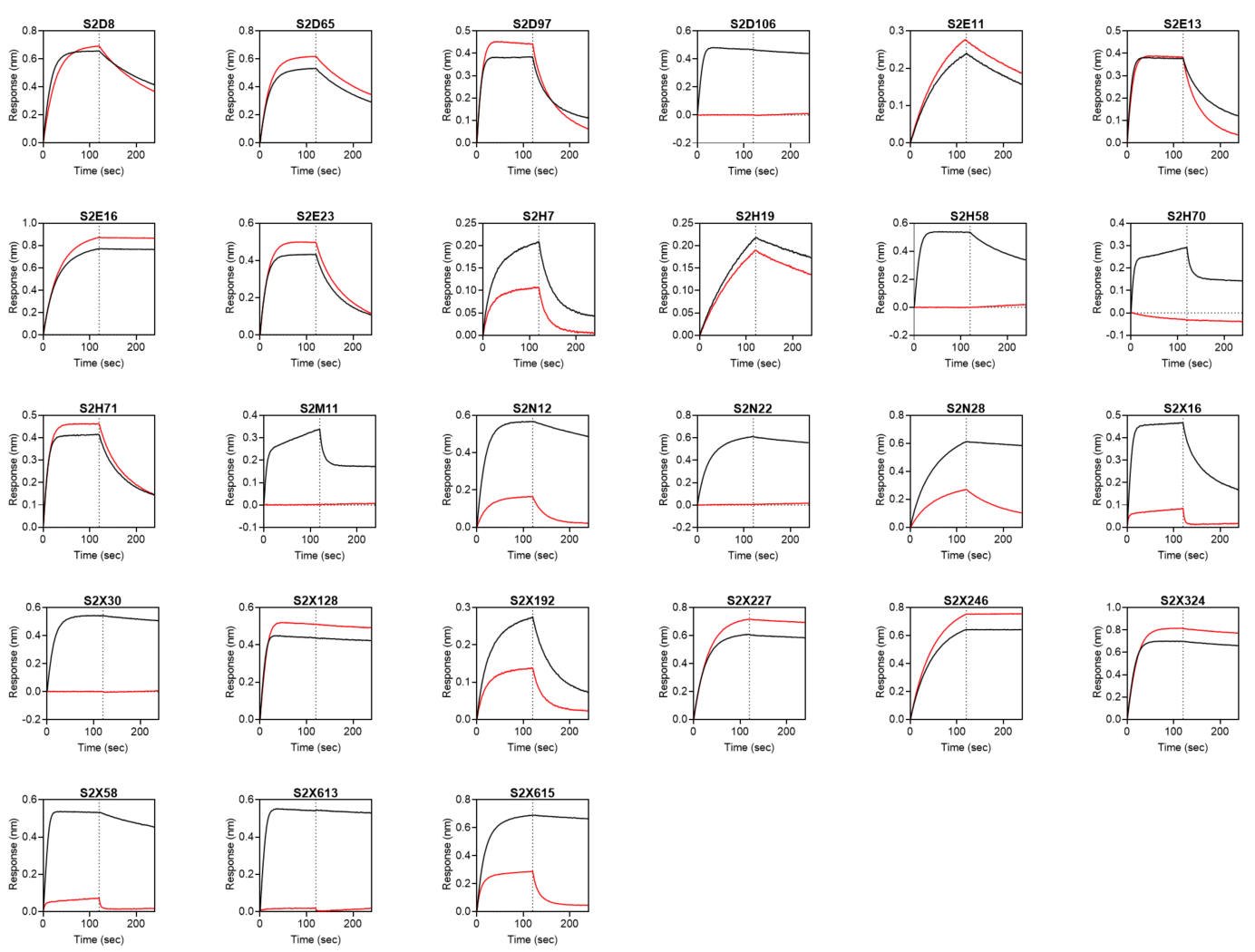

b
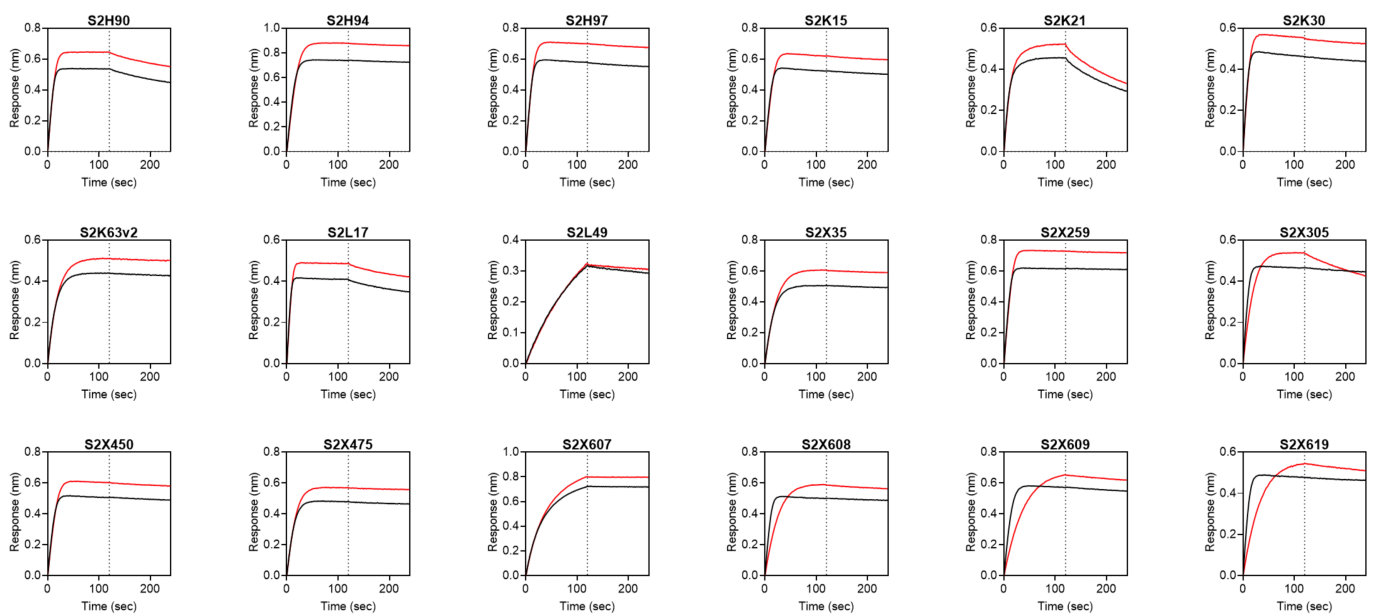

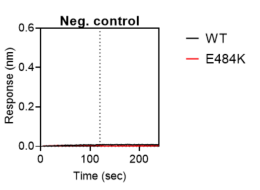

Extended Data Fig. 8 | Kinetics of binding to the RBD of wild-type and spike(E484K) SARS-CoV-2 for 46 RBD-specific monoclonal antibodies. a, b, Binding to the RBD of wild-type (black) and spike(E484K) (red) SARS-CoV-2 by 27 RBM-targeting (a) and 19 non-RBM-targeting (b) monoclonal antibodies. An antibody of irrelevant specificity was included as a negative control. 


\section{Article}

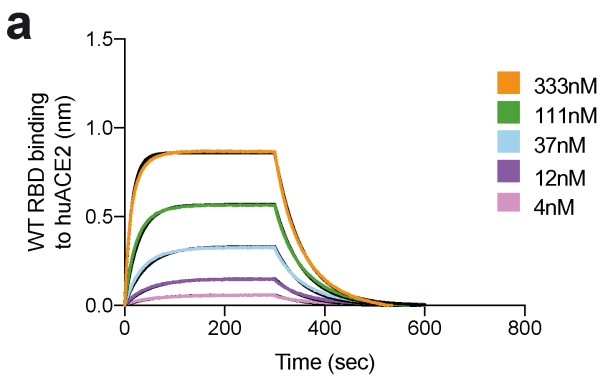

b
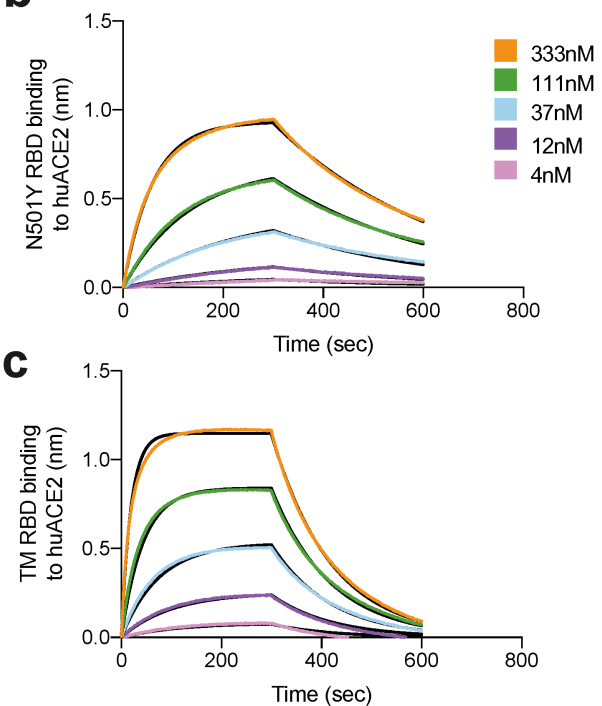

Extended Data Fig. 9 | Binding of human ACE2 to the RBDs of the wild-type SARS-CoV-2 spike, spike(N501Y) and spike(N501Y, E484K, K417N) proteins.

a-c, Biolayer interferometry binding analysis of the human ACE2 (huACE2) ectodomain (residues 1-615) to immobilized RBD of wild-type SARS-CoV-2 (a) and the RBD of B.1.1.7 spike(N501Y) (b) and spike (N501Y, E484K, K417N) proteins (c). Black lines correspond to a global fit of the data using a 1:1 binding model. 
Extended Data Table 1 | Kinetic analysis of human ACE2 binding to RBDs of SARS-CoV-2

\begin{tabular}{|c|c|c|c|c|}
\hline & & SARS-CoV-2 RBD WT & SARS-CoV-2 RBD N501Y & SARS-CoV-2 RBD TM \\
\hline KD (nM) & & 133 & 22 & 64 \\
Kon $\left(\mathbf{M}^{-1} \cdot \mathbf{s}^{-1}\right)$ & \multirow{2}{*}{ hACE2 } & $1.3^{*} 10^{5}$ & $1.4^{*} 10^{5}$ & $1.3^{*} 10^{5}$ \\
$\mathbf{K o f f}\left(\mathbf{s}^{-1}\right)$ & & $1.8^{*} 10^{-2}$ & $3 * 10^{-3}$ & $8.5^{*} 10^{-3}$ \\
\hline
\end{tabular}

Kinetic analyses were carried out using biolayer interferometry for the RBDs of the Wuhan-1 spike, spike(N501Y) and spike(N501Y, E484K, K417N) (TM) proteins. Values reported represent the global fit to the data shown in Extended Data Fig. 9. 


\section{Reporting Summary}

Nature Research wishes to improve the reproducibility of the work that we publish. This form provides structure for consistency and transparency in reporting. For further information on Nature Research policies, see our Editorial Policies and the Editorial Policy Checklist.

\section{Statistics}

For all statistical analyses, confirm that the following items are present in the figure legend, table legend, main text, or Methods section.

n/a Confirmed

$\square$ The exact sample size $(n)$ for each experimental group/condition, given as a discrete number and unit of measurement

$\square$ \ A statement on whether measurements were taken from distinct samples or whether the same sample was measured repeatedly

$\square$ The statistical test(s) used AND whether they are one- or two-sided

$\square$ Only common tests should be described solely by name; describe more complex techniques in the Methods section.

Х $\square$ A description of all covariates tested

Х $\square$ A description of any assumptions or corrections, such as tests of normality and adjustment for multiple comparisons

$\triangle$ A full description of the statistical parameters including central tendency (e.g. means) or other basic estimates (e.g. regression coefficient)

$\bigotimes$ AND variation (e.g. standard deviation) or associated estimates of uncertainty (e.g. confidence intervals)

$\varnothing$ For null hypothesis testing, the test statistic (e.g. $F, t, r$ ) with confidence intervals, effect sizes, degrees of freedom and $P$ value noted Give $P$ values as exact values whenever suitable.

\ $\square$ For Bayesian analysis, information on the choice of priors and Markov chain Monte Carlo settings

Х $\square$ For hierarchical and complex designs, identification of the appropriate level for tests and full reporting of outcomes

Х $\square$ Estimates of effect sizes (e.g. Cohen's $d$, Pearson's $r$ ), indicating how they were calculated

Our web collection on statistics for biologists contains articles on many of the points above.

\section{Software and code}

Policy information about availability of computer code

Data collection Sequences were obtained from GISAID using the search parameters defined in the methods section. Monoclonal antibody binding data were collected with Octet RED96 system (FortéBio). Monoclonal antibody neutralization data (luminescence) were collected with Synergy H1 microplate reader (BioTek). Sera neutralising antibody data were read on a Glomax luminometer (Promega).

Data analysis Graphad Prism v9 for statistical analyses and to produce figures. Monoclonal antibody binding data were analyzed by Pall FortéBio/Sartorius analysis software (version 12.0). Stata V13 for correlation analyses. PyMol v1.4 (Schodinger) to produce figures. Software versions and parameters used for all software are reported in full in the methods section.

For manuscripts utilizing custom algorithms or software that are central to the research but not yet described in published literature, software must be made available to editors and reviewers. We strongly encourage code deposition in a community repository (e.g. GitHub). See the Nature Research guidelines for submitting code \& software for further information.

\section{Data}

Policy information about availability of data

All manuscripts must include a data availability statement. This statement should provide the following information, where applicable:

- Accession codes, unique identifiers, or web links for publicly available datasets

- A list of figures that have associated raw data

- A description of any restrictions on data availability

The data analysed during the current study are available freely online in the GISAID database (https//gisaid.org) though specific files may be requested from the corresponding author on reasonable request. Pymol structures were all obtained from PDB and are available using the accession numbers described in the methods. 


\section{Field-specific reporting}

Please select the one below that is the best fit for your research. If you are not sure, read the appropriate sections before making your selection. \Life sciences

\section{Life sciences study design}

All studies must disclose on these points even when the disclosure is negative.

Sample size $n=37$. No sample size calculation was performed. The sample size of this study is sufficient to obtain a relevant analysis.
Data exclusions
Ne exclusions.
Replication performed 2 independent experiments and presented representative data with technical replicates. All data were reproducible.
Randomization is not relevant to the study as it is not an interventional study.
Blinding No blinding undertaken as this is not an interventional study.

\section{Reporting for specific materials, systems and methods}

We require information from authors about some types of materials, experimental systems and methods used in many studies. Here, indicate whether each material, system or method listed is relevant to your study. If you are not sure if a list item applies to your research, read the appropriate section before selecting a response.

Materials \& experimental systems

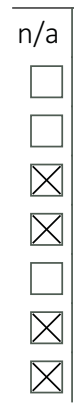
n/a Involved in the study

$\bigotimes$ Antibodies

$\square$ Eukaryotic cell lines

Х Palaeontology and archaeology

\ $\square$ Animals and other organisms

$\square$ Human research participants

$\bigotimes \square$ Clinical data

$\bigotimes \mid \square$ Dual use research of concern

\section{Antibodies}

\section{Antibodies used}

Validation

\begin{tabular}{l|l}
\multicolumn{2}{l}{ Methods } \\
\hline n/a & Involved in the study \\
$\square$ & $\square$ ChIP-seq \\
$\searrow$ & $\square$ Flow cytometry \\
$\searrow$ & $\square$ MRI-based neuroimaging
\end{tabular}

Х $\square$ MRI-based neuroimaging

\section{Eukaryotic cell lines}

Policy information about cell lines

Cell line source(s)

Authentication

Mycoplasma contamination

Commonly misidentified lines (See ICLAC register)
The source of monoclonal antibodies used in this study is described in Extended Data Table 1 and in the Method session.

The monoclonal antibodies were validated by binding and neutralization assays as described in the references of Extended Data Table 1.

\section{lines}

HEK $293 \mathrm{~T}$ and ExpiCHO cells were used for transfection work to produce pseudoviruses and mAbs, respectively. No cell lines used were authenticated. No new cell lines were generated.

All cell lines used were tested (by PCR) and were mycoplasma free.

No commonly misidentified lines were used in this study. 
Policy information about studies involving human research participants

Population characteristics

Individuals receiving the Pfizer BNT162b2 mRNA vaccine were consented for the study. Median age was 62 years (IQR 47-84) and $35 \%$ were female.

Recruitment

Participants were consented into the COVID-19 cohort of the NIHR Bioresource. Consecutive individuals were enrolled without exclusion.

Ethics oversight

The study was approved by the East of England - Cambridge Central Research Ethics Committee (17/EE/0025).

Note that full information on the approval of the study protocol must also be provided in the manuscript. 